\title{
Photocontrollable Modulation of Frontier Molecular Orbital Energy Levels of Cyclopentenone-Based Diarylethenes
}

Andrey G. Lvov*,a,b, Martin Herder ${ }^{c}$, Lutz Grubert ${ }^{c}$, Stefan Hecht ${ }^{c, d, e}$, Valerii Z. Shirinian ${ }^{f}$

${ }^{a}$ A. E. Favorsky Irkutsk Institute of Chemistry, Siberian Branch of the Russian Academy of Sciences 1 Favorsky St., Irkutsk, 664033, Russian Federation E-mail:1vov-andre@yandex.ru

${ }^{b}$ Irkutsk National Research Technical University 83, Lermontov St., Irkutsk, 664074, Russian Federation

${ }^{c}$ Department of Chemistry and IRIS Adlershof, Humboldt-Universität zu Berlin, Brook-TaylorStr. 2, 12489 Berlin, Germany

${ }^{d}$ DWI-Leibniz Institute for Interactive Materials, Forckenbeckstr. 50, 52074 Aachen, Germany

${ }^{e}$ Institute of Technical and Macromolecular Chemistry, RWTH Aachen University, Worringer Weg 2, 52074 Aachen, Germany

${ }^{f}$ N. D. Zelinsky Institute of Organic Chemistry, Russian Academy of Sciences, 47, Leninsky prosp, 119991 Moscow, Russian Federation

\section{Table of Contents}

I. Cyclic Voltammetry $\mathrm{S} 2$

II. Analysis of Frontier Molecular Orbitals

III. Cartesian Coordinates of Optimized Structures 


\section{Cyclic Voltammetry}

Figure S1. Cyclic voltammetry in oxidation (top) and reduction (bottom) regions for open-ring (black line) and closed-ring (red line) isomers of 2 . Acetonitrile / $0.1 \mathrm{M} \mathrm{Bu}_{4} \mathrm{NPF}_{6}$ (scan rate $1 \mathrm{~V} / \mathrm{s}$ ).
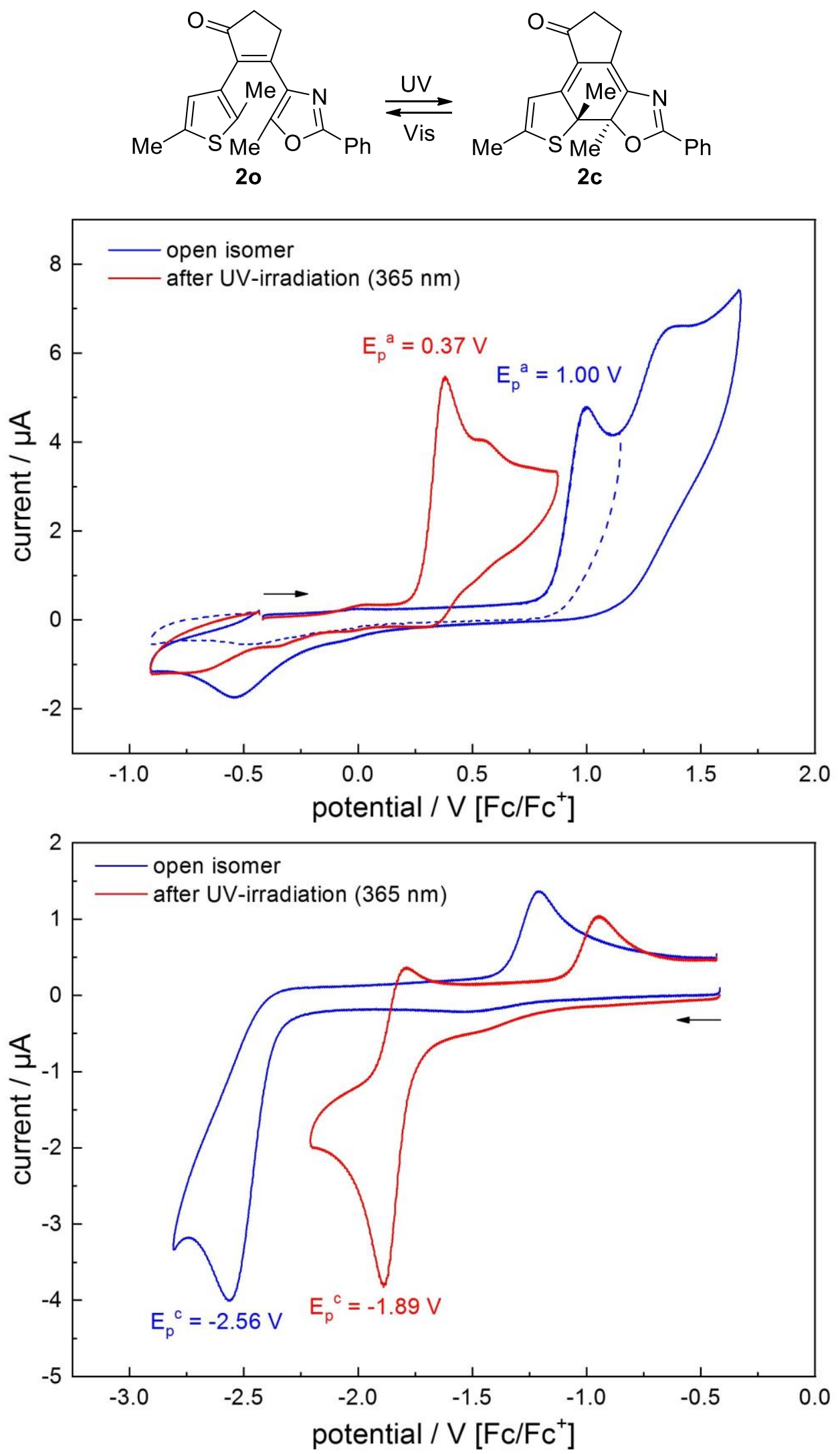
Figure S2. Cyclic voltammetry in oxidation (top) and reduction (bottom) regions for open-ring (black line) and closed-ring (red line) isomers of 3 . Acetonitrile / $0.1 \mathrm{M} \mathrm{Bu}_{4} \mathrm{NPF}_{6}$ (scan rate $1 \mathrm{~V} / \mathrm{s}$ ).
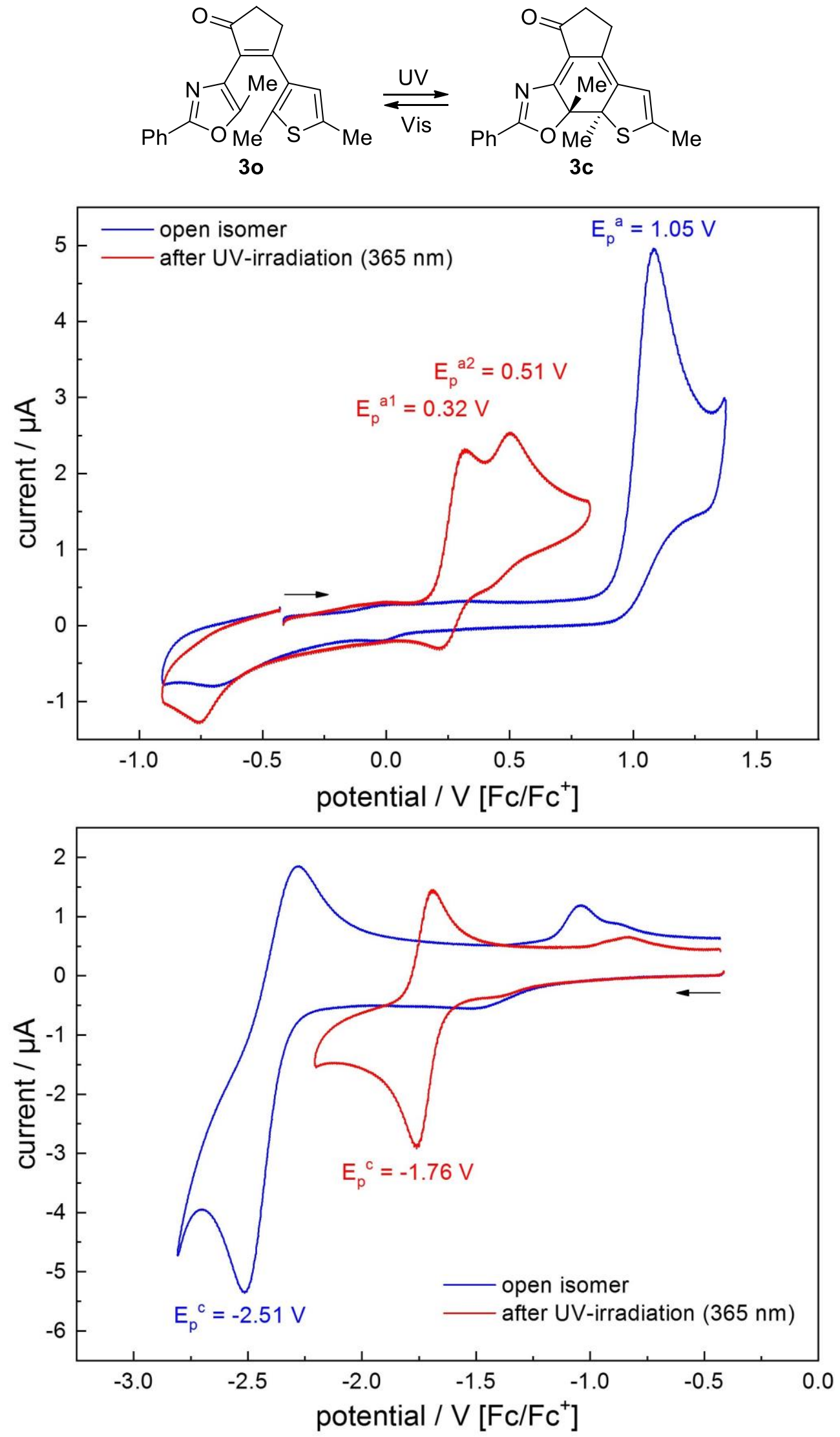
Figure S3. Cyclic voltammetry of open-ring isomer 3o during irradiation with UV light (365 nm), showing formation of closed-ring isomer $\mathbf{3 c}$.

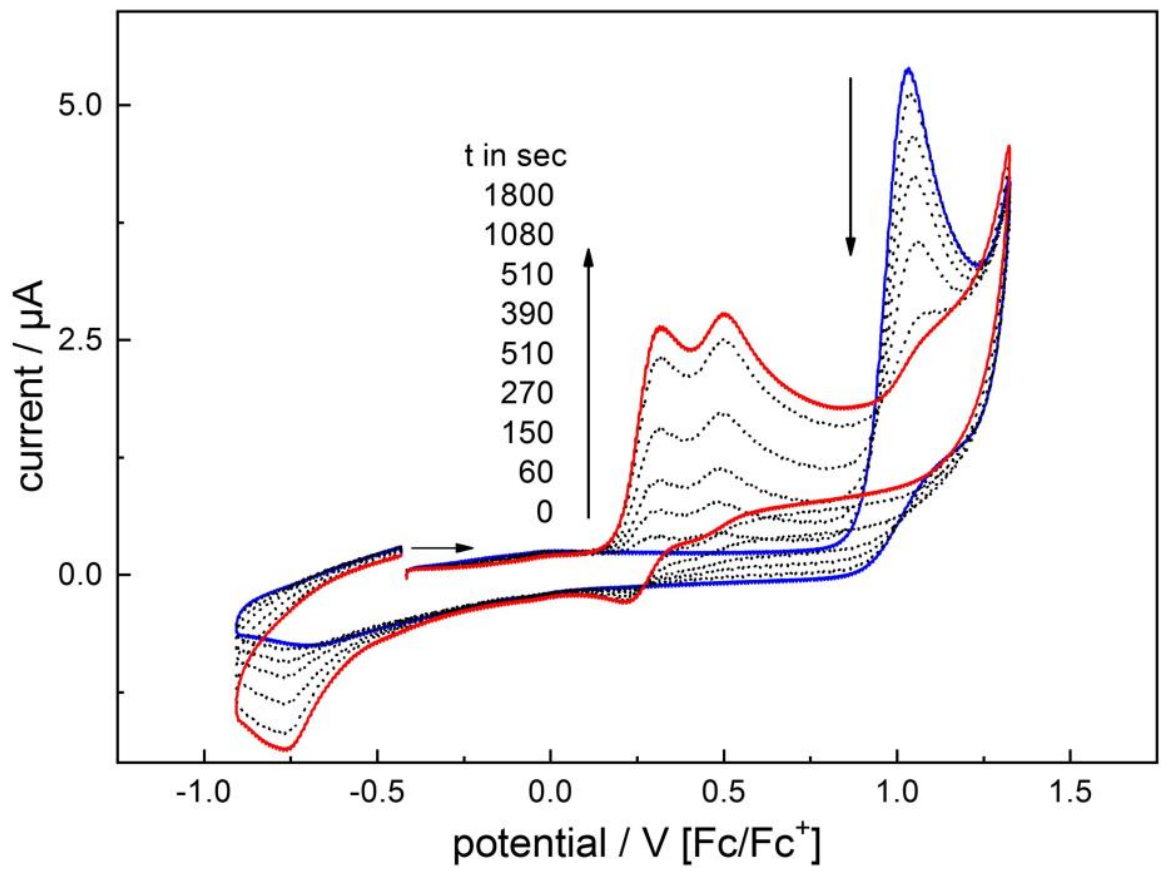

Figure S4. Cyclic voltammetry of closed-ring isomer $\mathbf{3 c}$ at varying scan rates demonstrating a quasireversible reduction at $\mathrm{E}_{\mathrm{p}}{ }^{\mathrm{c}}=-1.76 \mathrm{~V}$ and formation of side product formation.

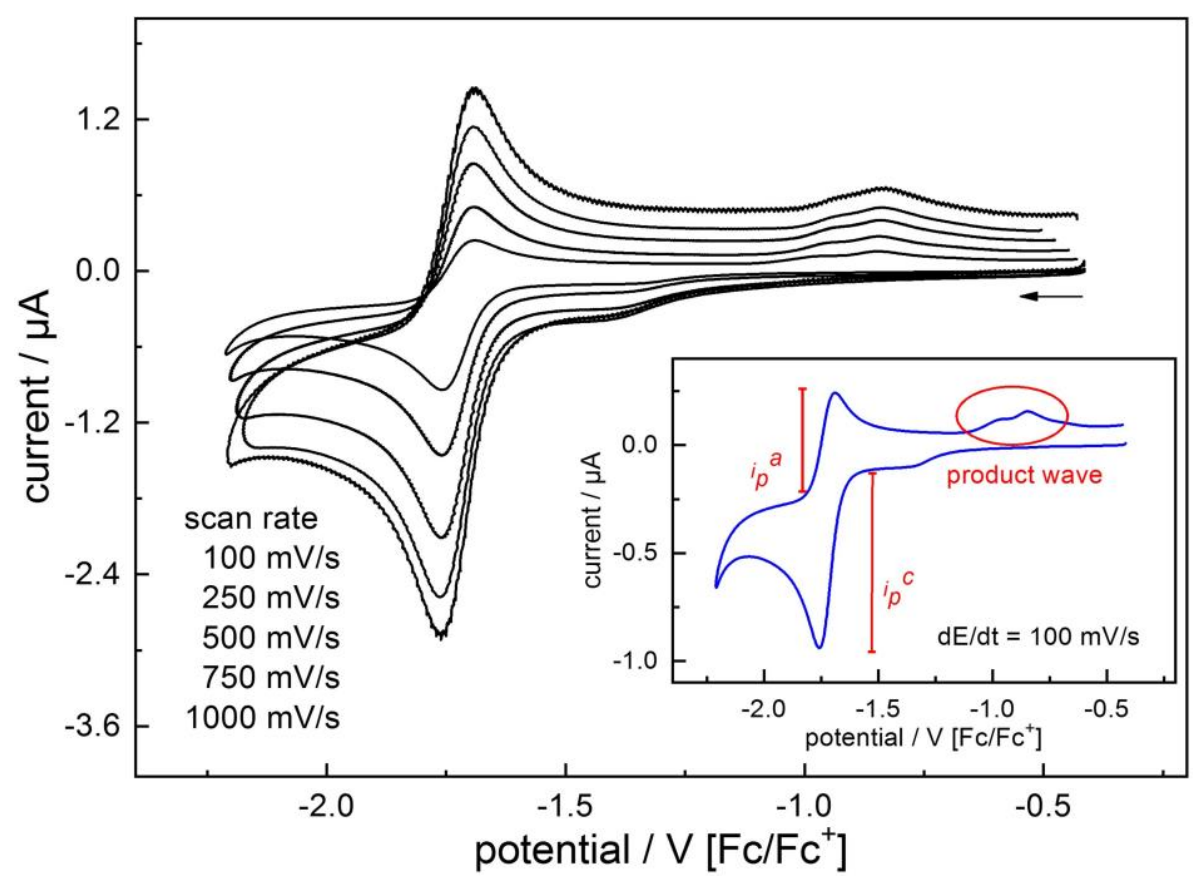


Figure S5. Cyclic voltammetry in oxidation (top) and reduction (bottom) regions for open-ring (black line) and closed-ring (red line) isomers of 4. Acetonitrile / $0.1 \mathrm{M} \mathrm{Bu}_{4} \mathrm{NPF}_{6}$ (scan rate $1 \mathrm{~V} / \mathrm{s}$ ).
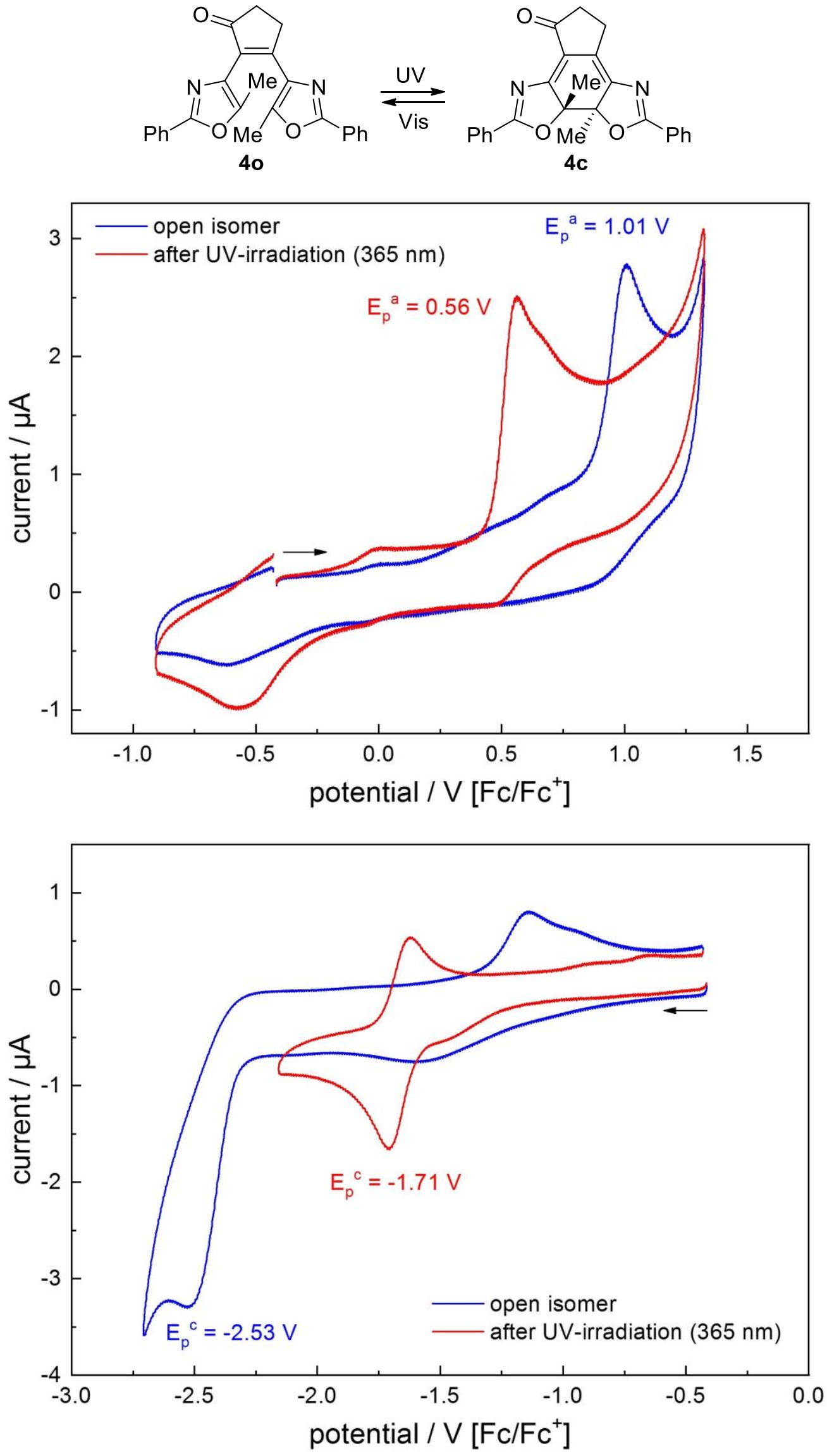
Figure S6. Cyclic voltammetry in oxidation (top) and reduction (bottom) regions for open-ring (black line) and closed-ring (red line) isomers of $\mathbf{5}$. Acetonitrile / $0.1 \mathrm{M} \mathrm{Bu}_{4} \mathrm{NPF}_{6}$ (scan rate $1 \mathrm{~V} / \mathrm{s}$ ).
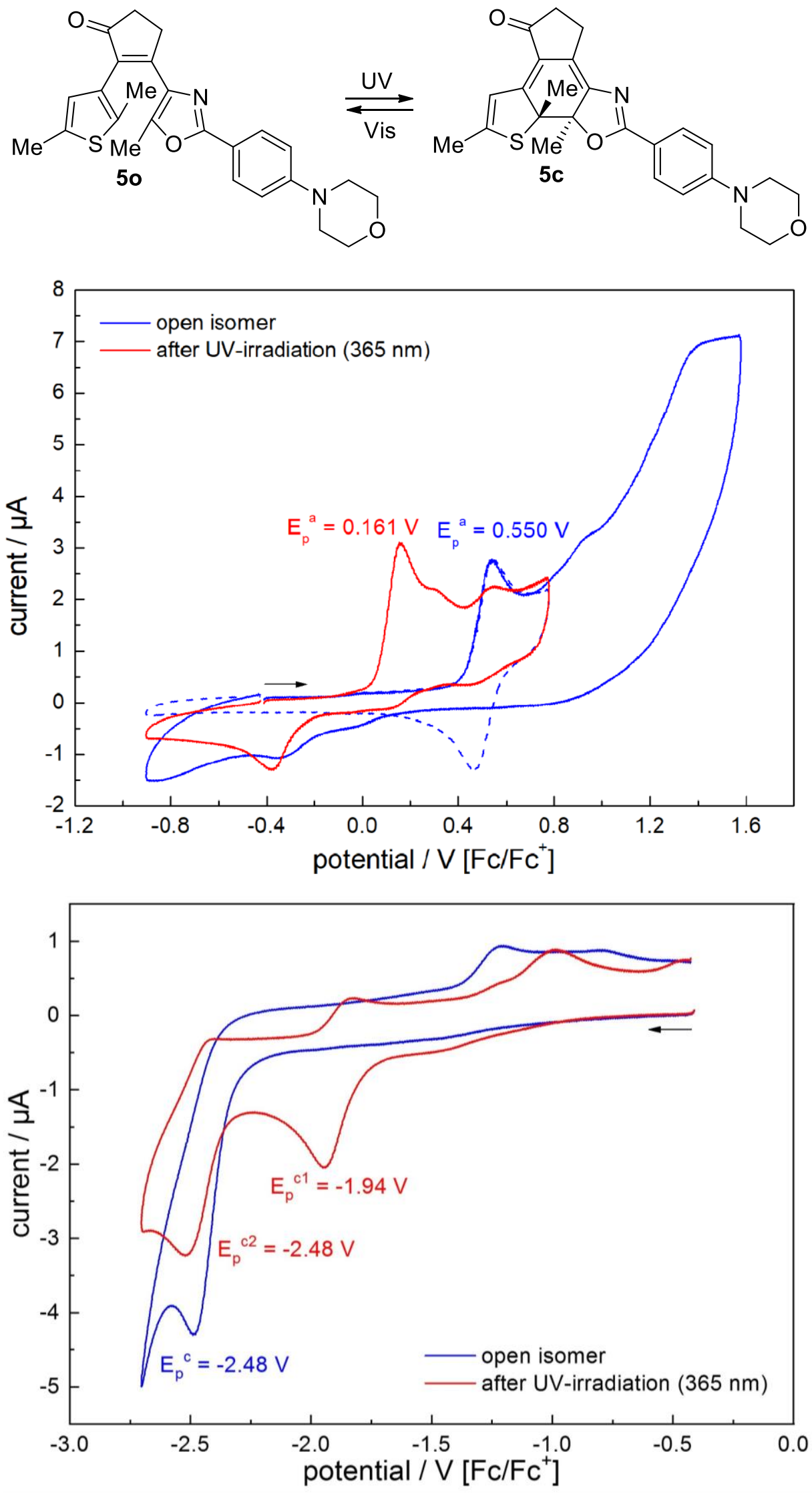
Figure S7. Cyclic voltammetry of open-ring isomer $\mathbf{5 0}$ at varying scan rates demonstrating a reversible oxidation at $\mathrm{E}_{\mathrm{p}}{ }^{\mathrm{a}}=0.55 \mathrm{~V}$.

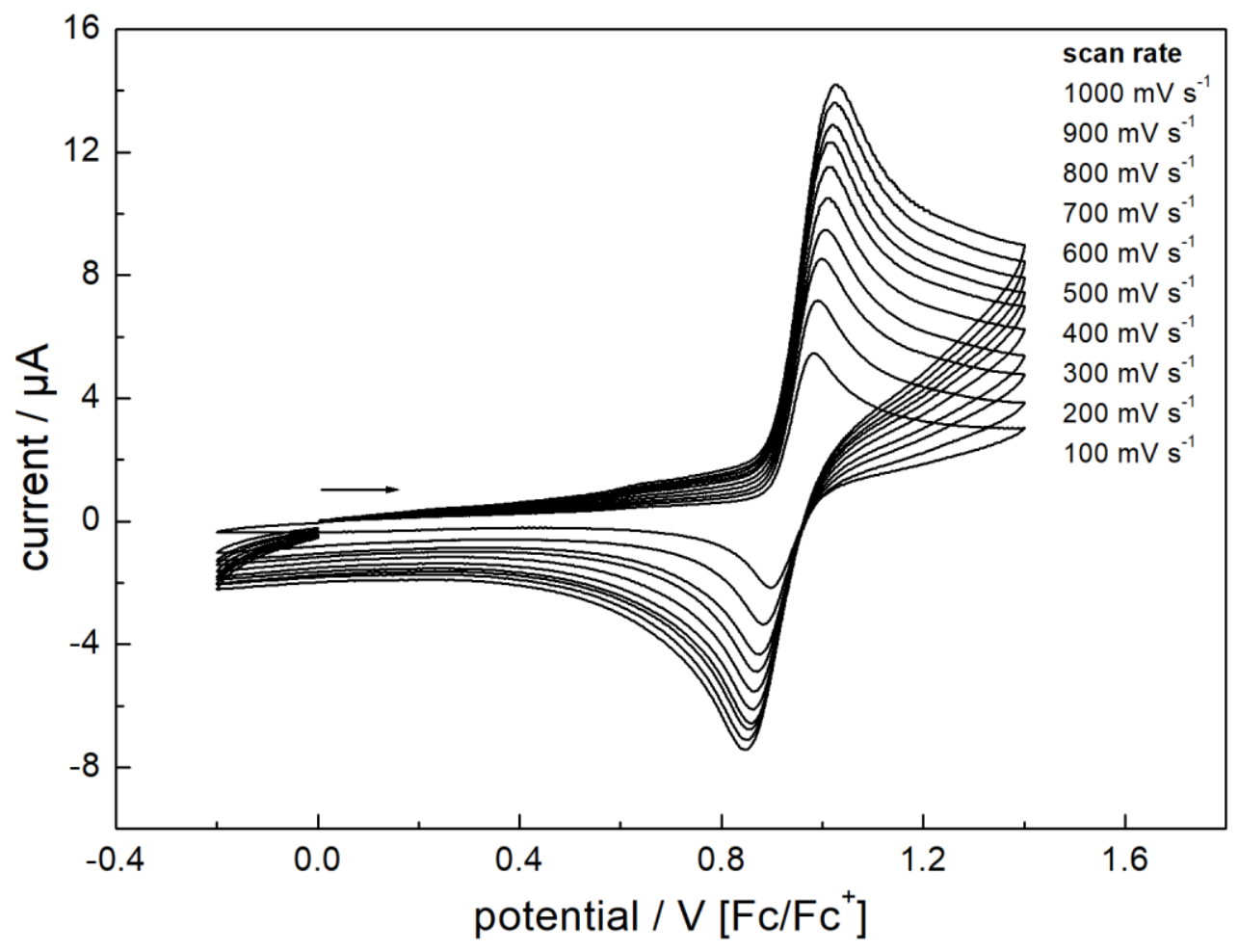

Figure S8. Irreversible character of oxidation of closed-ring isomer $\mathbf{5 c}$.

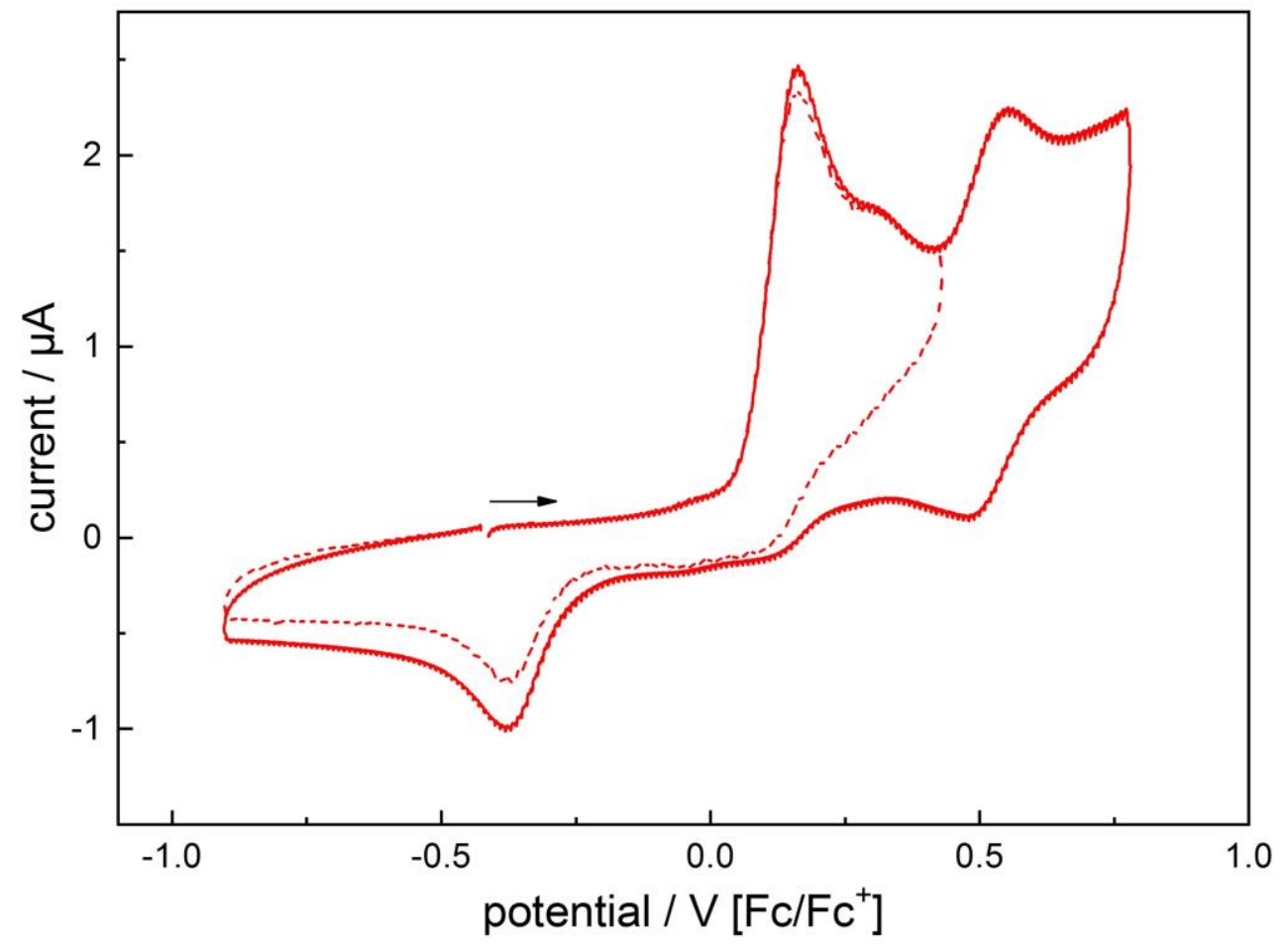


Figure S9. Irreversible character of reduction of closed-ring isomer $\mathbf{5 c}$.

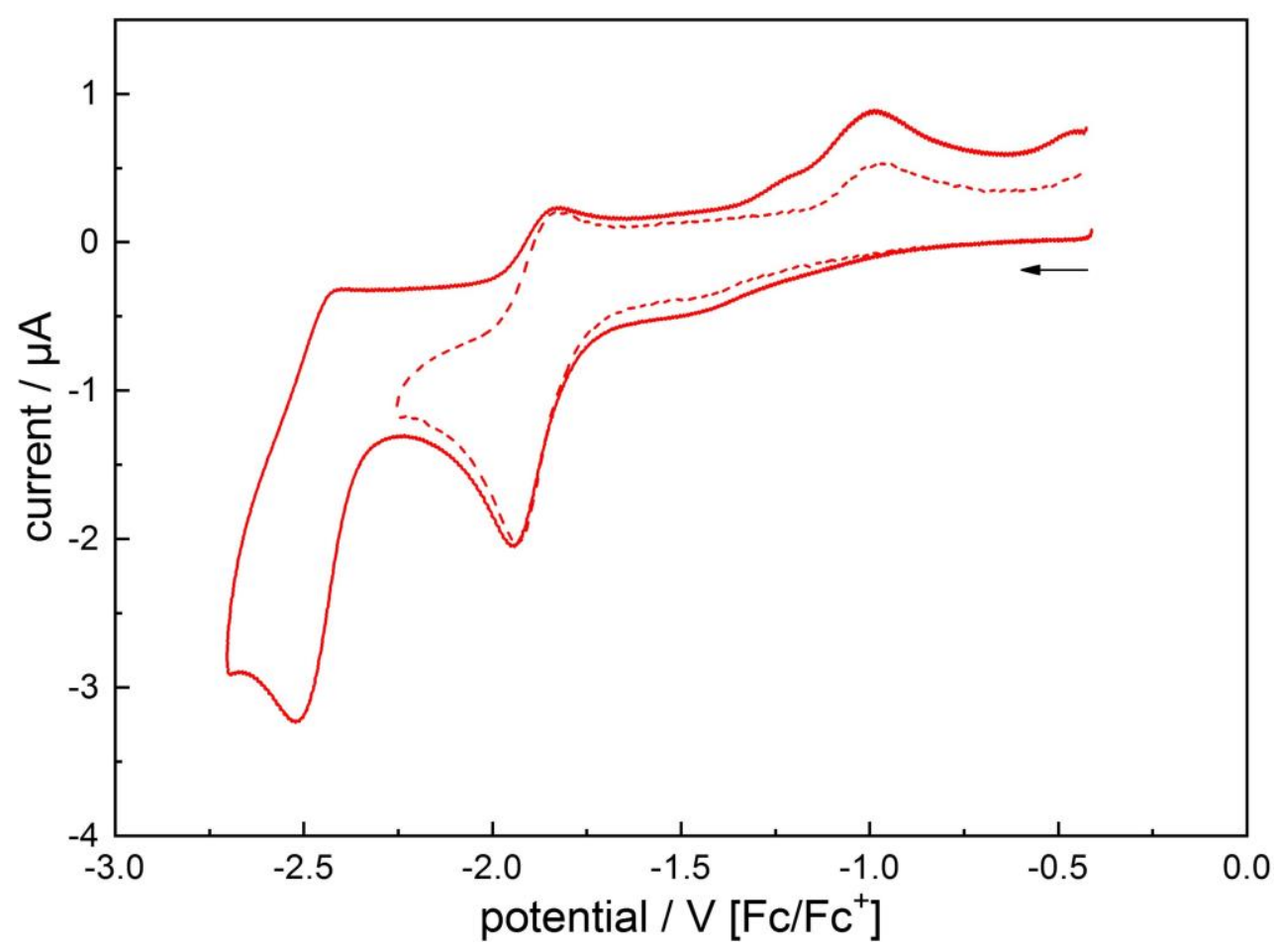

Figure S10. Cyclic voltammetry of open-ring isomer 5a during irradiation with UV light (365 $\mathrm{nm}$ ), showing formation of closed-ring isomer $\mathbf{5 c}$.

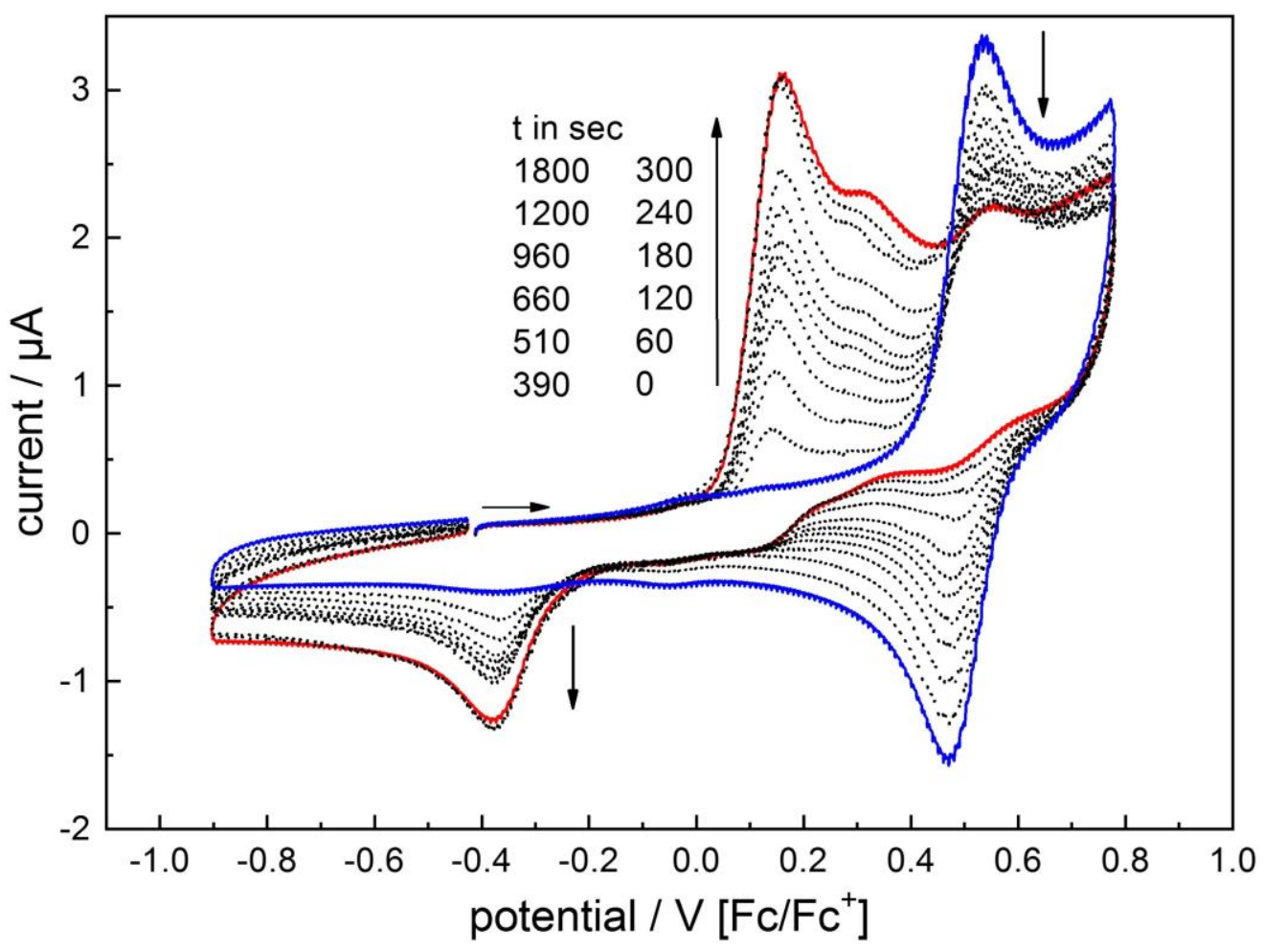


Figure S11. Cyclic voltammetry in oxidation (top) and reduction (bottom) regions for open-ring (black line) and closed-ring (red line) isomers of 6o. Acetonitrile / $0.1 \mathrm{M} \mathrm{Bu}_{4} \mathrm{NPF}_{6}$ (scan rate 1 $\mathrm{V} / \mathrm{s})$.

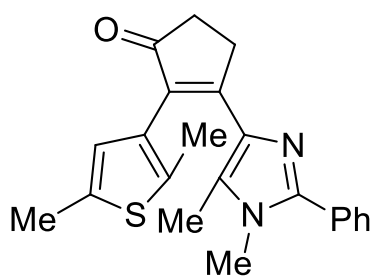

60

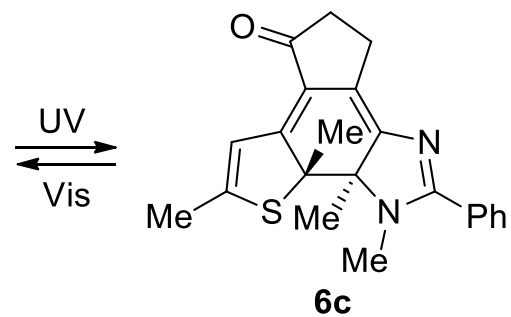

6c
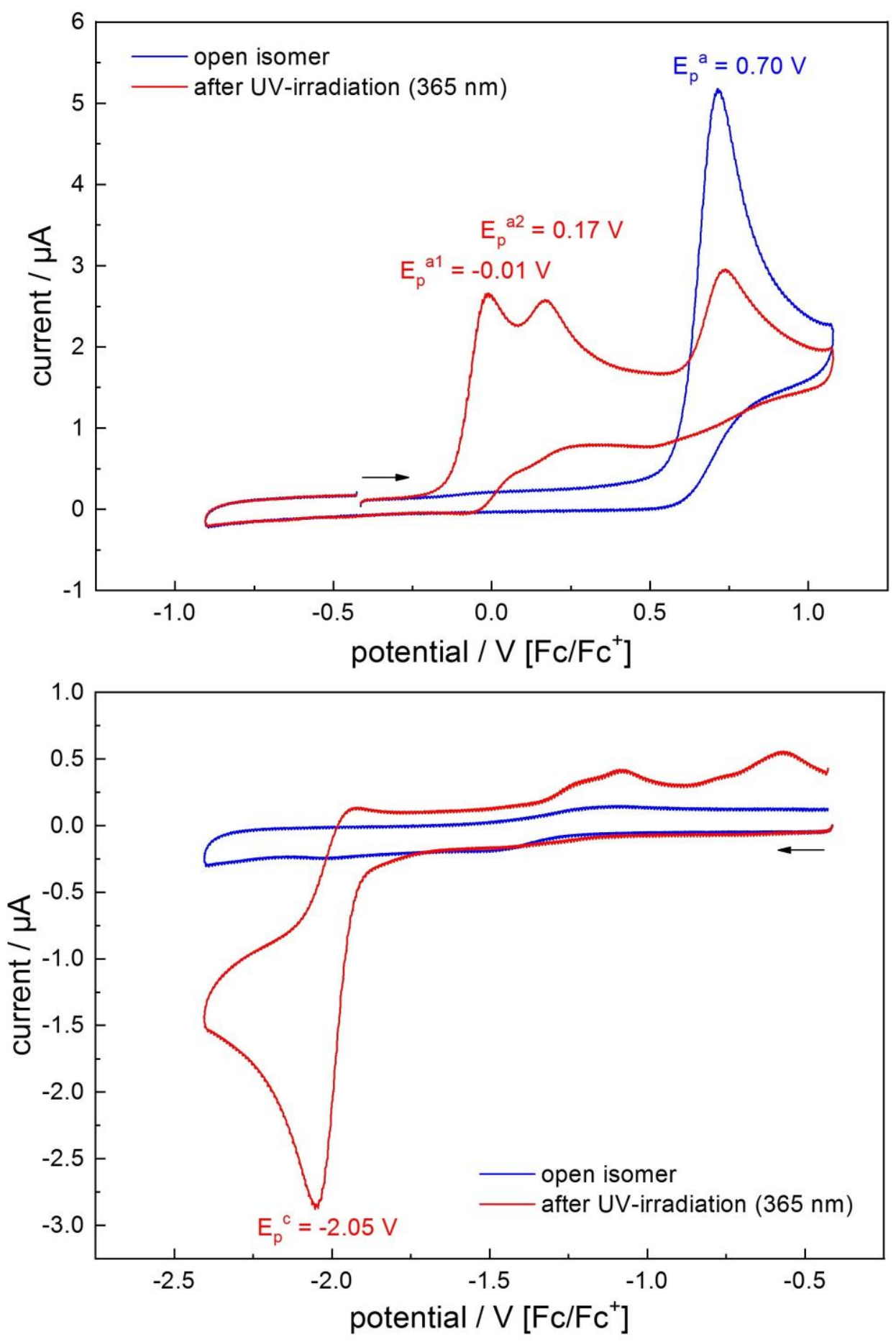
Figure S12. Cyclic voltammetry (oxidation region) of open-ring isomer 60 during irradiation with UV light (365 nm), showing formation of closed-ring isomer $\mathbf{6 c}$.

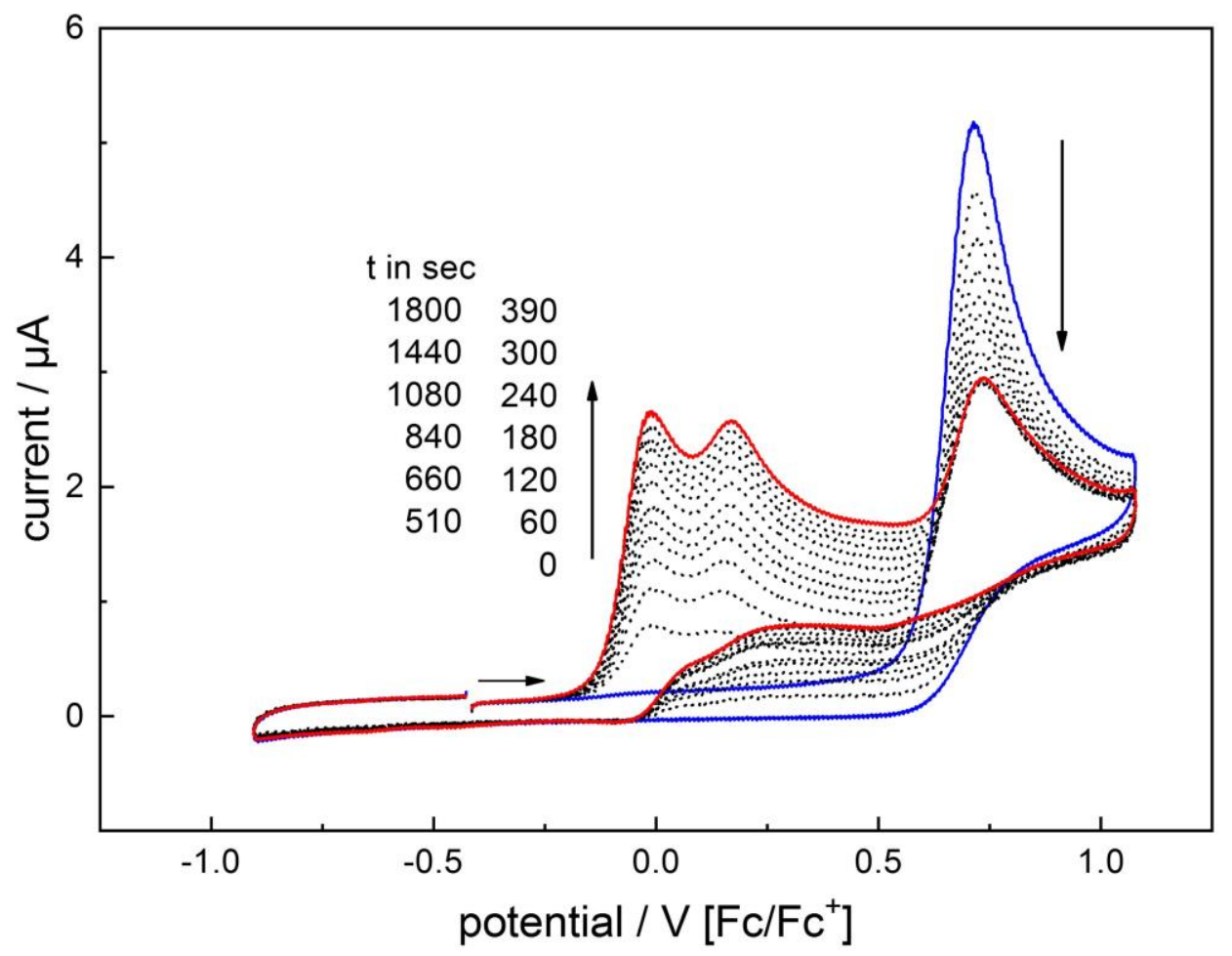

Figure S13. Cyclic voltammetry (reduction region) of open-ring isomer 60 during irradiation with UV light (365 nm), showing formation of closed-ring isomer $\mathbf{6 c}$.

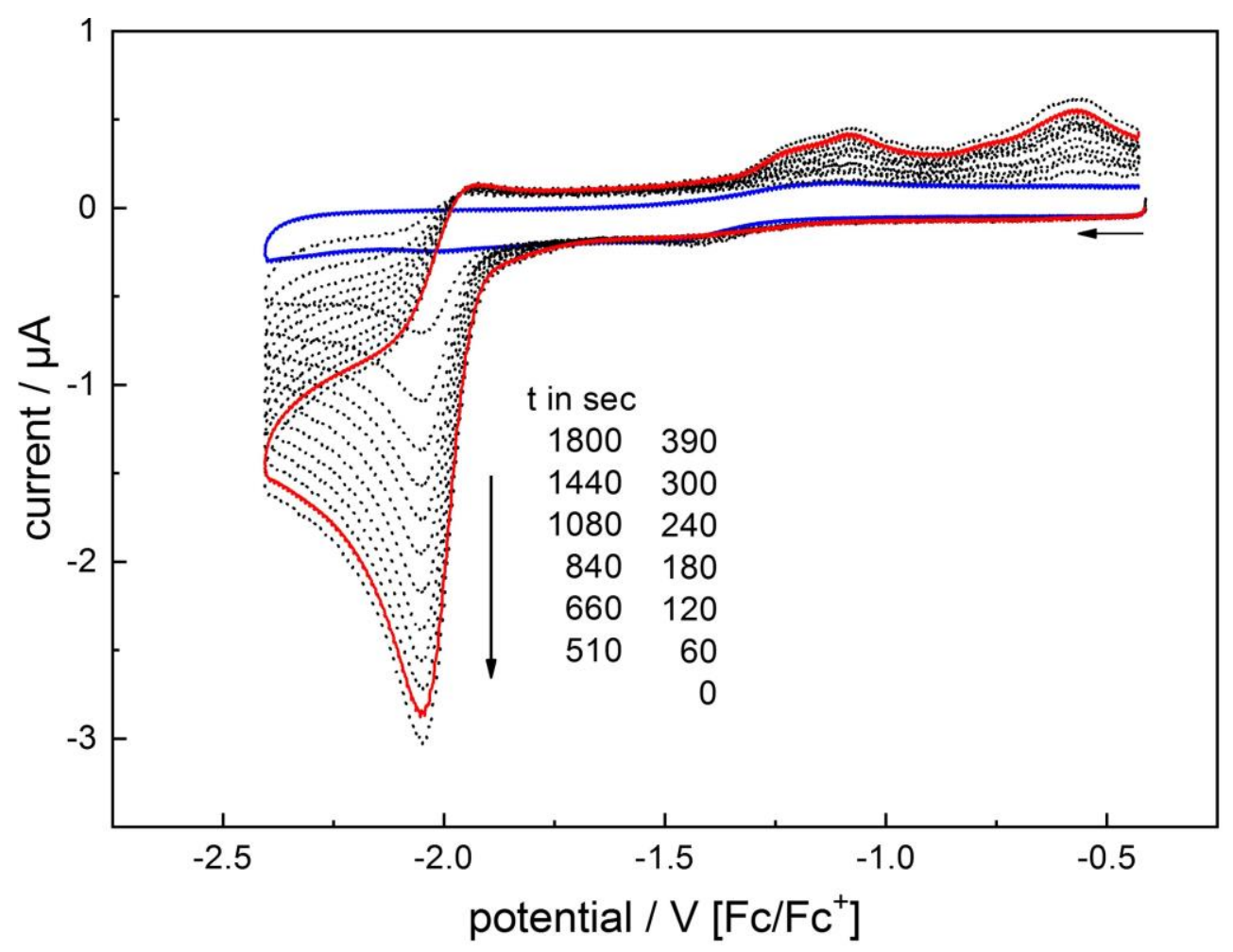




\section{Analysis of Frontier Molecular Orbitals}

Table S1. Molecular orbitals of 10 and 1c (isovalue: 0.05) according to DFT/B3LYP/6$311+\mathrm{G}(\mathrm{d}, \mathrm{p}) / \mathrm{PCM}(\mathrm{MeCN})$ level of theory.

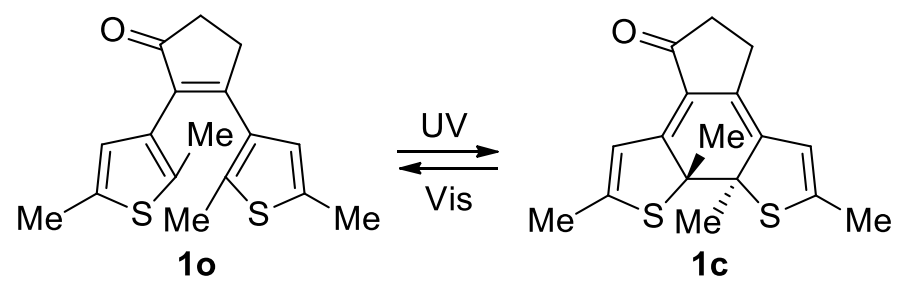

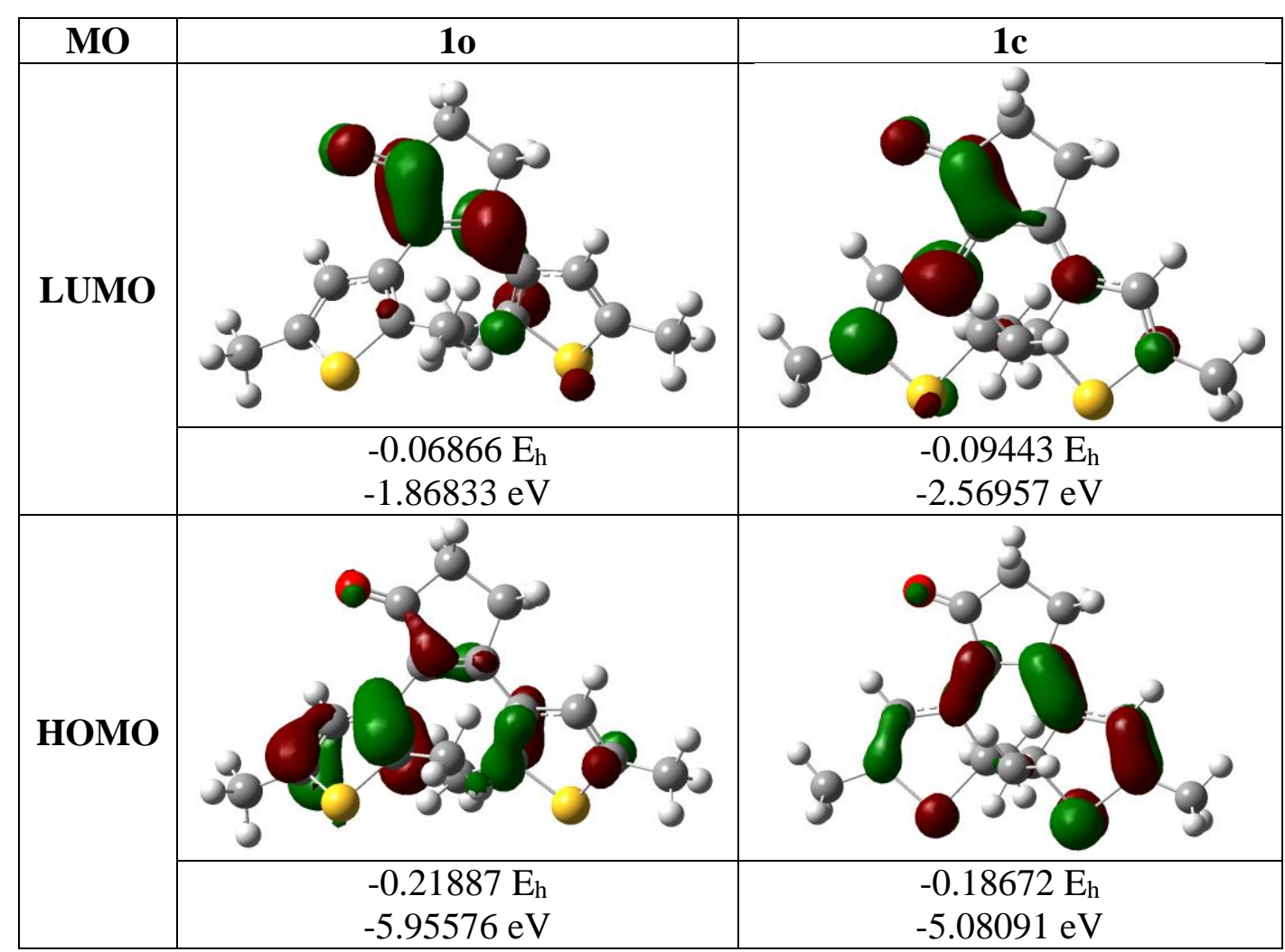


Table S2. Molecular orbitals of 20 and 2c (isovalue: 0.05) according to DFT/B3LYP/6$311+\mathrm{G}(\mathrm{d}, \mathrm{p}) / \mathrm{PCM}(\mathrm{MeCN})$ level of theory.

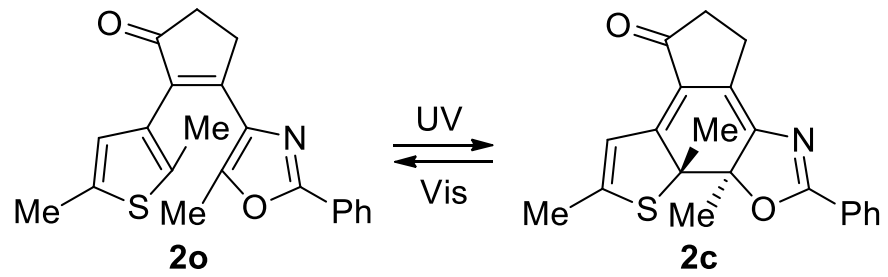

LUMO


Table S3. Molecular orbitals of 30 and 3c (isovalue: 0.05) according to DFT/B3LYP/6$311+\mathrm{G}(\mathrm{d}, \mathrm{p}) / \mathrm{PCM}(\mathrm{MeCN})$ level of theory.
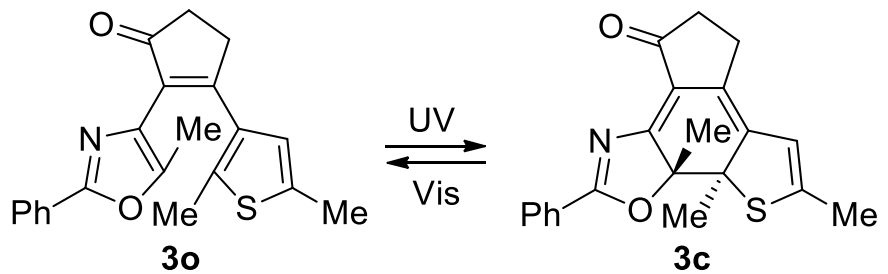

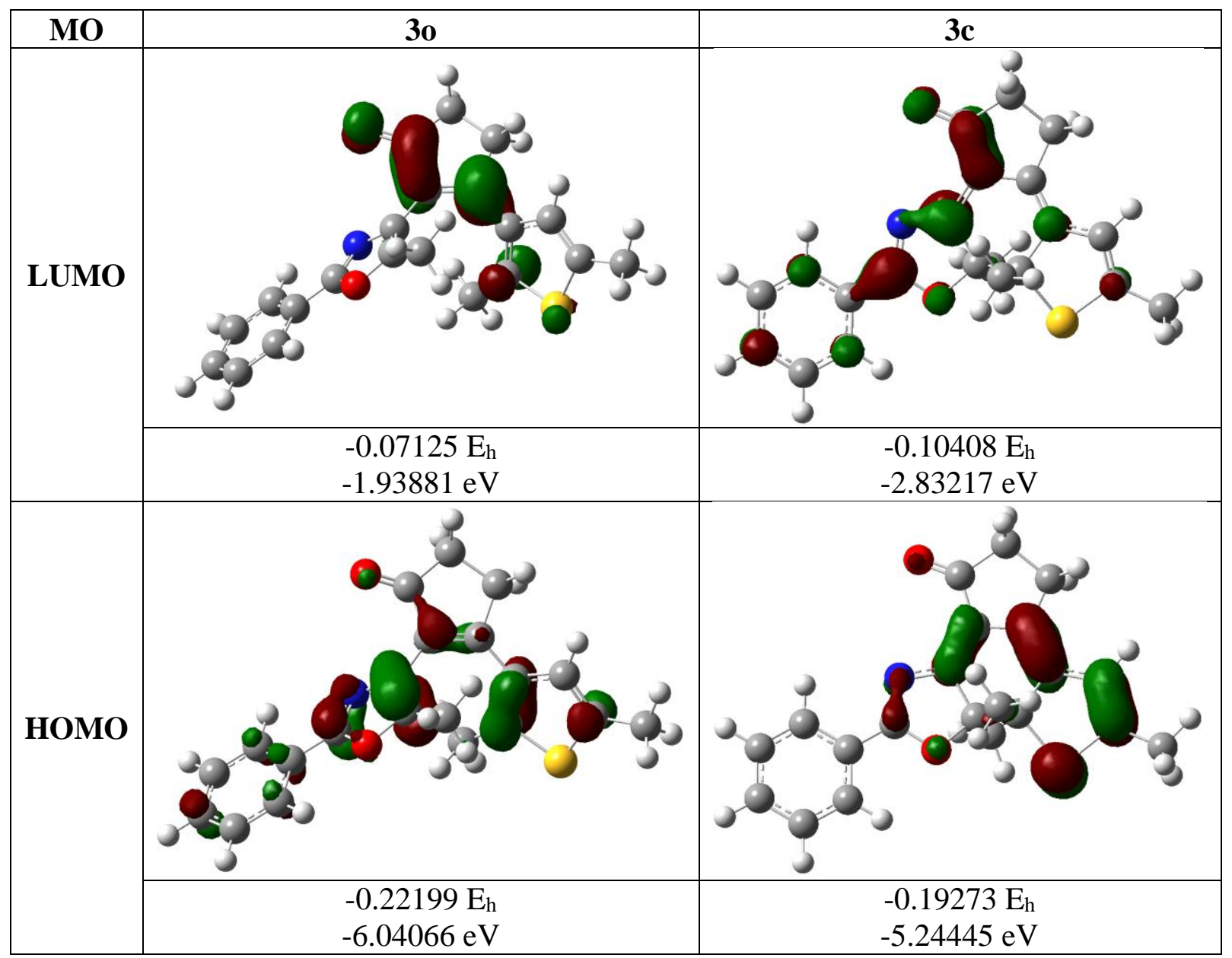


Table S4. Molecular orbitals of 40 and 4c (isovalue: 0.05) according to DFT/B3LYP/6$311+\mathrm{G}(\mathrm{d}, \mathrm{p}) / \mathrm{PCM}(\mathrm{MeCN})$ level of theory.
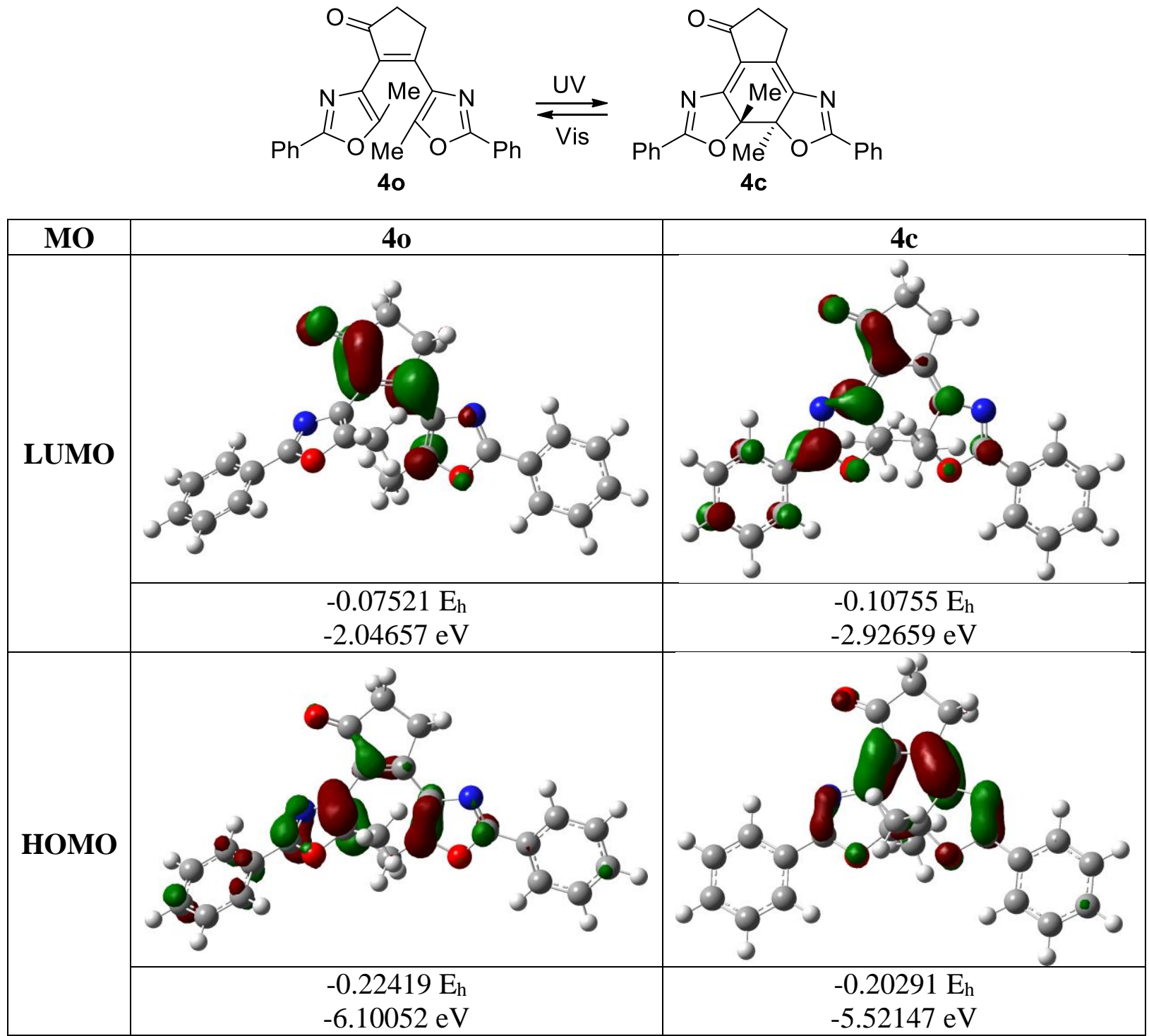
Table S5. Molecular orbitals of 50 and 5c (isovalue: 0.05) according to DFT/B3LYP/6$311+\mathrm{G}(\mathrm{d}, \mathrm{p}) / \mathrm{PCM}(\mathrm{MeCN})$ level of theory.

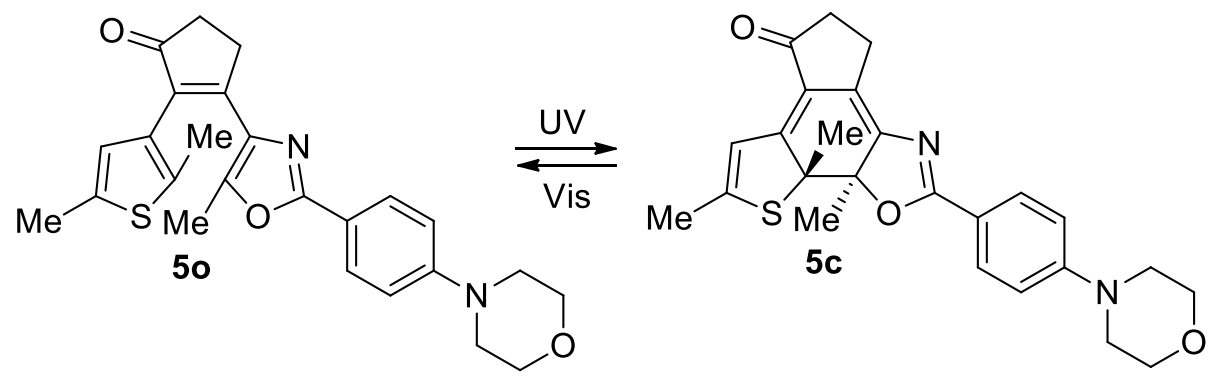

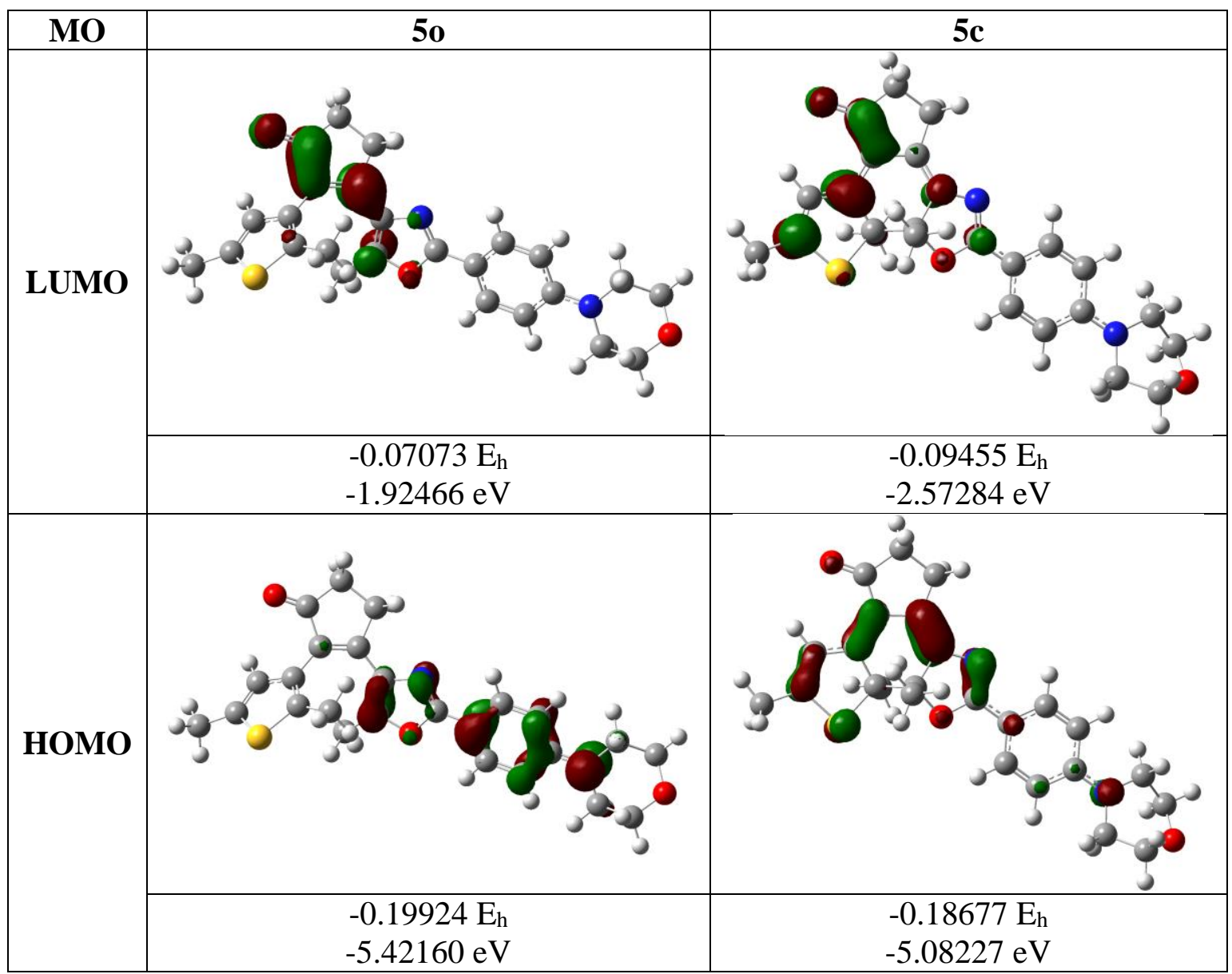


Table S6. Molecular orbitals of $\mathbf{6 0}$ and 6c (isovalue: 0.05) according to DFT/B3LYP/6$311+\mathrm{G}(\mathrm{d}, \mathrm{p}) / \mathrm{PCM}(\mathrm{MeCN})$ level of theory.
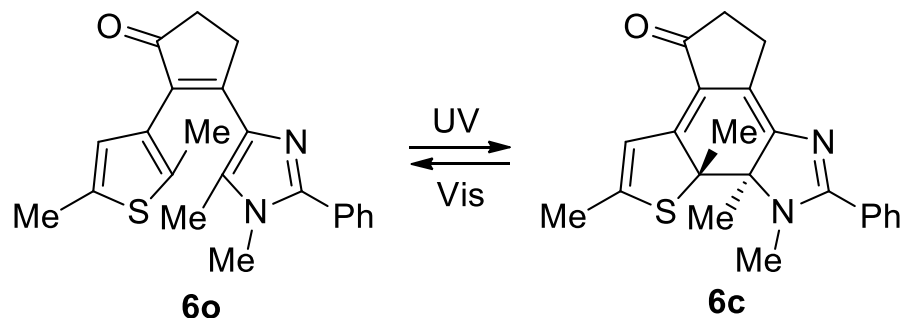

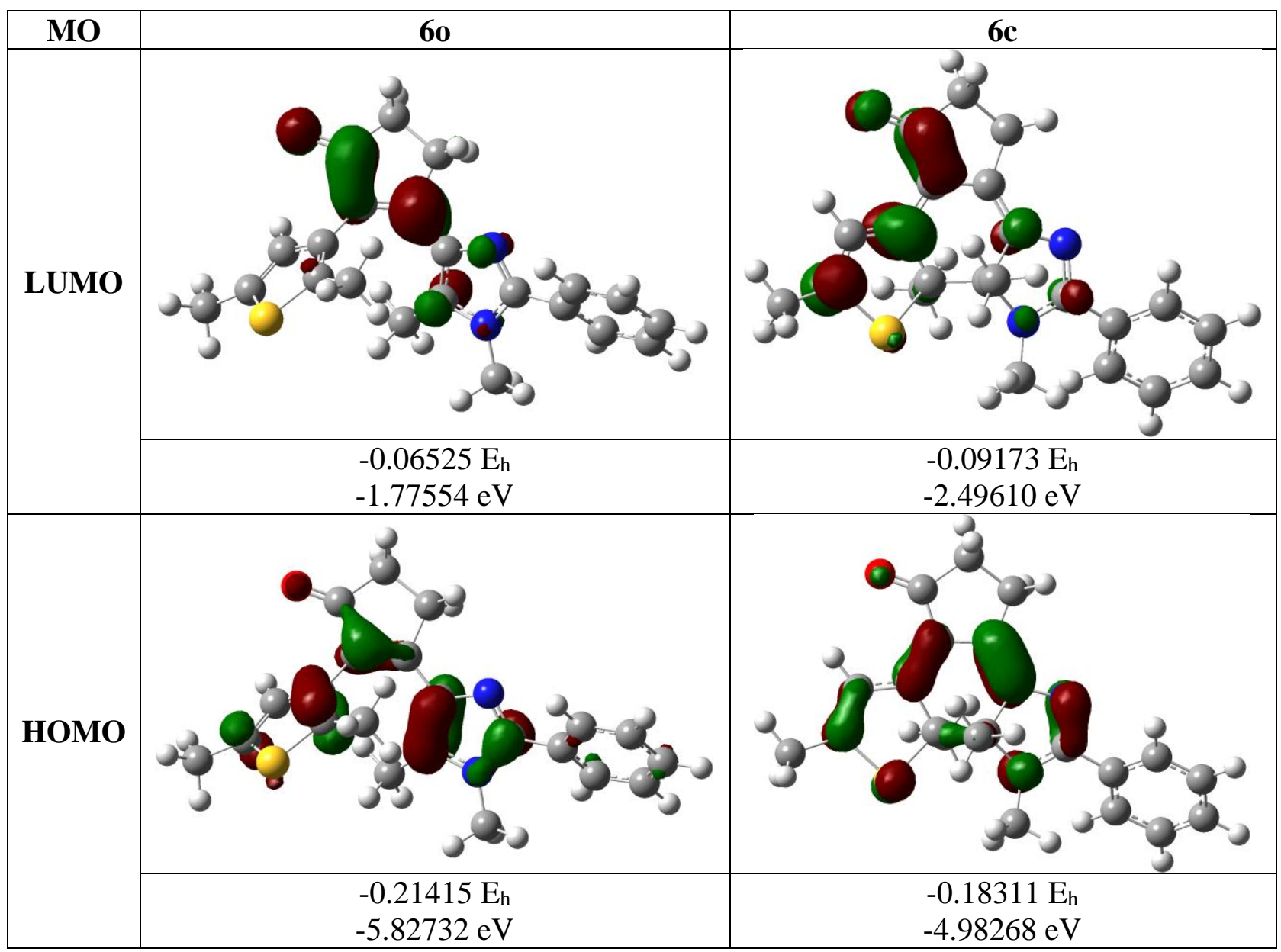




\section{Cartesian Coordinates of Optimized Structures}

Cartesian coordinate columns of the optimized structure for compound 10 (B3LYP/6-311+G(d,p)/PCM(acetonitrile))

$\begin{array}{lllll}1 & \mathrm{C} & -1.2609910 & 2.7492780 & 0.0064860 \\ 2 & \mathrm{C} & -0.1314220 & 3.7577260 & -0.1366360 \\ 3 & \mathrm{C} & -0.6799520 & 1.3867680 & 0.0039210 \\ 4 & \mathrm{C} & 1.1508300 & 2.9183960 & -0.1375290 \\ 5 & \mathrm{C} & 0.6761040 & 1.4766430 & -0.1128230 \\ 6 & \mathrm{C} & -1.5348570 & 0.1993030 & 0.1772480 \\ 7 & \mathrm{C} & -1.3612120 & -0.7593440 & 1.1471570 \\ 8 & \mathrm{C} & -2.6927460 & -0.0472740 & -0.6439050 \\ 9 & \mathrm{~S} & -2.6115030 & -1.9785830 & 1.0287540 \\ 10 & \mathrm{C} & -3.3776060 & -1.1816980 & -0.3307470 \\ 11 & \mathrm{C} & 1.6649260 & 0.4004200 & -0.2173510 \\ 12 & \mathrm{C} & 2.9130740 & 0.4416200 & 0.5064310 \\ 13 & \mathrm{C} & 1.5698590 & -0.7023760 & -1.0401210 \\ 14 & \mathrm{C} & 3.7373240 & -0.6161410 & 0.2735240 \\ 15 & \mathrm{~S} & 3.0028790 & -1.6895460 & -0.8991380 \\ 16 & \mathrm{O} & -2.4451610 & 3.0341850 & 0.1187710 \\ 17 & \mathrm{C} & -4.6051280 & -1.7472740 & -0.9780680 \\ 18 & \mathrm{C} & -0.3146660 & -0.8633390 & 2.2141520 \\ 19 & \mathrm{C} & 0.4988090 & -1.0947190 & -2.0110040 \\ 20 & \mathrm{C} & 5.0774770 & -0.9131140 & 0.8737660 \\ 21 & \mathrm{H} & -0.1831770 & 4.4834320 & 0.6782610 \\ 22 & \mathrm{H} & -0.2661800 & 4.3117070 & -1.0698280 \\ 23 & \mathrm{H} & 1.7770840 & 3.1275700 & 0.7353870 \\ 24 & \mathrm{H} & 1.7766640 & 3.0955910 & -1.0165820 \\ 25 & \mathrm{H} & -2.9902040 & 0.6082000 & -1.4525880 \\ 26 & \mathrm{H} & 3.1691600 & 1.2293760 & 1.2038050 \\ 27 & \mathrm{H} & -4.4251610 & -2.7441250 & -1.3915380 \\ 28 & \mathrm{H} & -5.4365110 & -1.8305580 & -0.2716910 \\ 29 & \mathrm{H} & -4.9217320 & -1.0958360 & -1.7952040 \\ 30 & \mathrm{H} & 0.0961050 & 0.1228530 & 2.4379490 \\ 31 & \mathrm{H} & -0.7267000 & -1.2769590 & 3.1376830 \\ 32 & \mathrm{H} & 0.5170180 & -1.5038370 & 1.9032360 \\ 33 & \mathrm{H} & 0.9222600 & -1.6008630 & -2.8814640 \\ 34 & \mathrm{H} & -0.0426840 & -0.2141910 & -2.3598920 \\ 35 & \mathrm{H} & -0.2311580 & -1.7688950 & -1.5529090 \\ 36 & \mathrm{H} & 5.8554200 & -0.9958090 & 0.1092100 \\ 37 & \mathrm{H} & 5.3596980 & -0.1099630 & 1.5572400 \\ 38 & \mathrm{H} & 5.0704310 & -1.8503510 & 1.4378960\end{array}$


Cartesian coordinate columns of the optimized structure for compound 1c

(B3LYP/6-311+G(d,p)/PCM(acetonitrile))

$\begin{array}{lllll}1 & \mathrm{C} & 1.7265160 & 2.7415680 & -0.0441060 \\ 2 & \mathrm{C} & 0.7293330 & 3.9001590 & -0.1078660 \\ 3 & \mathrm{C} & 0.9579600 & 1.4952620 & 0.0438670 \\ 4 & \mathrm{C} & -0.6612080 & 3.2969660 & 0.1974800 \\ 5 & \mathrm{C} & -0.4642320 & 1.8001510 & 0.0468810 \\ 6 & \mathrm{C} & 1.4197260 & 0.2085000 & 0.1314480 \\ 7 & \mathrm{C} & 0.3843170 & -0.8868500 & 0.4256860 \\ 8 & \mathrm{C} & 2.7450460 & -0.3003980 & 0.0202710 \\ 9 & \mathrm{~S} & 1.2715710 & -2.4807330 & -0.0084340 \\ 10 & \mathrm{C} & 2.8307770 & -1.6573740 & -0.0656120 \\ 11 & \mathrm{C} & -1.3837360 & 0.8189490 & -0.1005210 \\ 12 & \mathrm{C} & -2.8217540 & 0.8888980 & -0.0505280 \\ 13 & \mathrm{C} & -0.8886110 & -0.6093730 & -0.4067500 \\ 14 & \mathrm{C} & -3.4592270 & -0.3009850 & 0.0241730 \\ 15 & \mathrm{~S} & -2.3514440 & -1.6989980 & 0.0392380 \\ 16 & \mathrm{O} & 2.9456990 & 2.8673510 & -0.0703360 \\ 17 & \mathrm{C} & 4.0751400 & -2.4689380 & -0.2124180 \\ 18 & \mathrm{C} & 0.1403390 & -0.9051550 & 1.9562730 \\ 19 & \mathrm{C} & -0.6656180 & -0.7340280 & -1.9314780 \\ 20 & \mathrm{C} & -4.9297030 & -0.5500620 & 0.1094190 \\ 21 & \mathrm{H} & 1.0291900 & 4.6952250 & 0.5774940 \\ 22 & \mathrm{H} & 0.7730720 & 4.3159590 & -1.1205190 \\ 23 & \mathrm{H} & -0.9645440 & 3.5377770 & 1.2222160 \\ 24 & \mathrm{H} & -1.4382500 & 3.6826920 & -0.4651550 \\ 25 & \mathrm{H} & 3.6111240 & 0.3442260 & -0.0428780 \\ 26 & \mathrm{H} & -3.3613220 & 1.8288080 & -0.0300530 \\ 27 & \mathrm{H} & 4.2001410 & -3.1458860 & 0.6384940 \\ 28 & \mathrm{H} & 4.0368400 & -3.0886340 & -1.1135140 \\ 29 & \mathrm{H} & 4.9490640 & -1.8190920 & -0.2724010 \\ 30 & \mathrm{H} & 1.0922780 & -1.0253600 & 2.4750200 \\ 31 & \mathrm{H} & -0.3151380 & 0.0334450 & 2.2771980 \\ 32 & \mathrm{H} & -0.5193690 & -1.7252860 & 2.2367560 \\ 33 & \mathrm{H} & 0.1439570 & -0.0740880 & -2.2533800 \\ 34 & \mathrm{H} & -0.4179120 & -1.7557080 & -2.2187450 \\ 35 & \mathrm{H} & -1.5768050 & -0.4365390 & -2.4522730 \\ 36 & \mathrm{H} & -5.1836450 & -1.1237550 & 1.0066540 \\ 37 & \mathrm{H} & -5.4733530 & 0.3958870 & 0.1391830 \\ 38 & \mathrm{H} & -5.2833240 & -1.1265220 & -0.7515920\end{array}$


Cartesian coordinate columns of the optimized structure for compound $\mathbf{2 0}$

(B3LYP/6-311+G(d,p)/PCM(acetonitrile))

$\begin{array}{lllll}1 & \mathrm{C} & -2.6065990 & 2.6225320 & 0.0057970 \\ 2 & \mathrm{C} & -1.6089810 & 3.7528450 & -0.1954660 \\ 3 & \mathrm{C} & -1.8751280 & 1.3338300 & -0.0424790 \\ 4 & \mathrm{C} & -0.2416770 & 3.0665670 & -0.2867580 \\ 5 & \mathrm{C} & -0.5511090 & 1.5836280 & -0.2459880 \\ 6 & \mathrm{C} & -2.5756390 & 0.0562490 & 0.1765440 \\ 7 & \mathrm{C} & -2.2570640 & -0.8545480 & 1.1557010 \\ 8 & \mathrm{C} & -3.7247010 & -0.3390500 & -0.5970020 \\ 9 & \mathrm{~S} & -3.3611900 & -2.2118700 & 1.1057550 \\ 10 & \mathrm{C} & -4.2611100 & -1.5383610 & -0.2382240 \\ 11 & \mathrm{C} & 0.5438670 & 0.6406330 & -0.4212470 \\ 12 & \mathrm{~N} & 1.8269990 & 0.9604100 & 0.0333960 \\ 13 & \mathrm{C} & 0.5944620 & -0.5704620 & -1.0607210 \\ 14 & \mathrm{C} & 2.5766970 & -0.0412020 & -0.3065870 \\ 15 & \mathrm{O} & 1.8894460 & -1.0088710 & -0.9838430 \\ 16 & \mathrm{O} & -3.8061330 & 2.7668260 & 0.1915260 \\ 17 & \mathrm{C} & -1.1690320 & -0.8121780 & 2.1850090 \\ 18 & \mathrm{C} & -0.3530590 & -1.4204400 & -1.8183010 \\ 19 & \mathrm{C} & 4.0018100 & -0.2455780 & -0.0636490 \\ 20 & \mathrm{C} & 4.7382000 & 0.7404720 & 0.6113520 \\ 21 & \mathrm{C} & 4.6481290 & -1.4136310 & -0.4948530 \\ 22 & \mathrm{C} & 6.0962380 & 0.5583080 & 0.8478280 \\ 23 & \mathrm{C} & 6.0081280 & -1.5888630 & -0.2537570 \\ 24 & \mathrm{C} & 6.7361480 & -0.6058840 & 0.4167820 \\ 25 & \mathrm{C} & -5.4384170 & -2.2543660 & -0.8269820 \\ 26 & \mathrm{H} & -1.6904290 & 4.4638140 & 0.6298900 \\ 27 & \mathrm{H} & -1.8651360 & 4.2929540 & -1.1112490 \\ 28 & \mathrm{H} & 0.4166180 & 3.3312960 & 0.5453850 \\ 29 & \mathrm{H} & 0.3024220 & 3.3168970 & -1.2015940 \\ 30 & \mathrm{H} & -4.1256260 & 0.2600540 & -1.4048760 \\ 31 & \mathrm{H} & -0.8231940 & 0.2129460 & 2.3292390 \\ 32 & \mathrm{H} & -1.5171950 & -1.1908340 & 3.1490960 \\ 33 & \mathrm{H} & -0.3046510 & -1.4147320 & 1.8864600 \\ 34 & \mathrm{H} & 0.1215860 & -1.7867310 & -2.7319370 \\ 35 & \mathrm{H} & -1.2435620 & -0.8546670 & -2.0846830 \\ 36 & \mathrm{H} & -0.6664050 & -2.2876730 & -1.2285250 \\ 37 & \mathrm{H} & 4.2391360 & 1.6416580 & 0.9449270 \\ 38 & \mathrm{H} & 4.0873050 & -2.1794600 & -1.0155500 \\ 39 & \mathrm{H} & 6.6573470 & 1.3251240 & 1.3693470 \\ 40 & \mathrm{H} & 6.4992210 & -2.4945870 & -0.5902070 \\ 41 & \mathrm{H} & 7.7948120 & -0.7450790 & 0.6029090 \\ 43 & \mathrm{H} & -5.1632640 & -3.2389100 & -1.2166000 \\ 44 & \mathrm{H} & -6.2332940 & -2.4037970 & -0.0901390 \\ & & -5.8491420 & -1.6678670 & -1.6512150\end{array}$


Cartesian coordinate columns of the optimized structure for compound $\mathbf{2 c}$

(B3LYP/6-311+G(d,p)/PCM(acetonitrile))

$\begin{array}{lllll}1 & \mathrm{C} & -3.2233050 & 2.3397850 & -0.0425650 \\ 2 & \mathrm{C} & -2.5108140 & 3.6919240 & 0.0467450 \\ 3 & \mathrm{C} & -2.1939430 & 1.2919490 & -0.0426610 \\ 4 & \mathrm{C} & -1.0051450 & 3.4104110 & -0.1702270 \\ 5 & \mathrm{C} & -0.8790770 & 1.9166090 & 0.0329990 \\ 6 & \mathrm{C} & -2.3510280 & -0.0656000 & -0.1587160 \\ 7 & \mathrm{C} & -1.0864450 & -0.9245090 & -0.3508120 \\ 8 & \mathrm{C} & -3.5403340 & -0.8493840 & -0.1555620 \\ 9 & \mathrm{~S} & -1.6444040 & -2.6651880 & 0.0551330 \\ 10 & \mathrm{C} & -3.3414430 & -2.1930920 & -0.0491220 \\ 11 & \mathrm{C} & 0.1920830 & 1.1312840 & 0.2484580 \\ 12 & \mathrm{~N} & 1.5519080 & 1.4046920 & 0.1711260 \\ 13 & \mathrm{C} & 0.0167560 & -0.3598080 & 0.5513150 \\ 14 & \mathrm{C} & 2.1491780 & 0.2538300 & 0.1382820 \\ 15 & \mathrm{O} & 1.3496610 & -0.8540300 & 0.2006780 \\ 16 & \mathrm{O} & -4.4382580 & 2.1923550 & -0.0997220 \\ 17 & \mathrm{C} & -0.6942380 & -0.9042370 & -1.8492830 \\ 18 & \mathrm{C} & -0.1836620 & -0.6029910 & 2.0533880 \\ 19 & \mathrm{C} & -4.3923070 & -3.2520290 & 0.0010000 \\ 20 & \mathrm{C} & 3.5872520 & 0.0277300 & 0.0147680 \\ 21 & \mathrm{C} & 4.4607440 & 1.1230510 & -0.0886140 \\ 22 & \mathrm{C} & 4.1083650 & -1.2751360 & -0.0052030 \\ 23 & \mathrm{C} & 5.8291460 & 0.9146080 & -0.2078840 \\ 24 & \mathrm{C} & 5.4807700 & -1.4761350 & -0.1262830 \\ 25 & \mathrm{C} & 6.3434240 & -0.3848320 & -0.2275860 \\ 26 & \mathrm{H} & -2.9397400 & 4.3931380 & -0.6713700 \\ 27 & \mathrm{H} & -2.7015500 & 4.0980960 & 1.0462330 \\ 28 & \mathrm{H} & -0.7080380 & 3.6810220 & -1.1890690 \\ 29 & \mathrm{H} & -0.3667620 & 3.9752990 & 0.5107600 \\ 30 & \mathrm{H} & -4.5264370 & -0.4064010 & -0.1820190 \\ 31 & \mathrm{H} & -1.5298360 & -1.2595120 & -2.4526370 \\ 32 & \mathrm{H} & -0.4449200 & 0.1108770 & -2.1642230 \\ 33 & \mathrm{H} & 0.1677940 & -1.5475500 & -2.0254580 \\ 34 & \mathrm{H} & -1.1614040 & -0.2388020 & 2.3733840 \\ 35 & \mathrm{H} & -0.1113970 & -1.6649560 & 2.2883920 \\ 36 & \mathrm{H} & 0.5846190 & -0.0653780 & 2.6121990 \\ 37 & \mathrm{H} & -4.2955790 & -3.9346740 & -0.8491080 \\ 38 & \mathrm{H} & -4.3003080 & -3.8546730 & 0.9096110 \\ 39 & \mathrm{H} & -5.3864970 & -2.8042410 & -0.0222190 \\ 40 & \mathrm{H} & 4.0557120 & 2.1268600 & -0.0745930 \\ 41 & \mathrm{H} & 3.4386670 & -2.1212810 & 0.0756580 \\ 42 & \mathrm{H} & 6.4976120 & 1.7640710 & -0.2864920 \\ 44 & \mathrm{H} & 5.8765380 & -2.4849410 & -0.1404820 \\ & & 7.4115580 & -0.5439570 & -0.3208480\end{array}$


Cartesian coordinate columns of the optimized structure for compound $\mathbf{3 0}$ (B3LYP/6-311+G(d,p)/PCM(acetonitrile))

$\begin{array}{lllll}1 & \mathrm{C} & 0.6039160 & 3.1486810 & 0.0452580 \\ 2 & \mathrm{C} & 1.9520700 & 3.7483820 & -0.3263240 \\ 3 & \mathrm{C} & 0.7441980 & 1.6742560 & 0.0675120 \\ 4 & \mathrm{C} & 2.9152500 & 2.5570730 & -0.3794140 \\ 5 & \mathrm{C} & 2.0376830 & 1.3338330 & -0.1914880 \\ 6 & \mathrm{C} & -0.3793050 & 0.8038080 & 0.4277600 \\ 7 & \mathrm{C} & -0.4632760 & -0.1130810 & 1.4338640 \\ 8 & \mathrm{~N} & -1.6016220 & 0.8496300 & -0.2444830 \\ 9 & \mathrm{O} & -1.7272090 & -0.6506280 & 1.3863840 \\ 10 & \mathrm{C} & -2.3569940 & -0.0228810 & 0.3512960 \\ 11 & \mathrm{C} & 2.6354320 & -0.0001500 & -0.2953630 \\ 12 & \mathrm{C} & 3.9080110 & -0.3098600 & 0.3113960 \\ 13 & \mathrm{C} & 2.1233000 & -1.0582560 & -1.0171780 \\ 14 & \mathrm{C} & 4.3437670 & -1.5801820 & 0.0907540 \\ 15 & \mathrm{~S} & 3.1980120 & -2.4303210 & -0.9250390 \\ 16 & \mathrm{O} & -0.4033720 & 3.7897910 & 0.3032290 \\ 17 & \mathrm{C} & 0.4493110 & -0.6029590 & 2.4935870 \\ 18 & \mathrm{C} & 0.8879980 & -1.1465450 & -1.8598990 \\ 19 & \mathrm{C} & 5.5880780 & -2.2468560 & 0.5919820 \\ 20 & \mathrm{C} & -3.7381760 & -0.3905760 & 0.0517950 \\ 21 & \mathrm{C} & -4.3948650 & -1.3956960 & 0.7776160 \\ 22 & \mathrm{C} & -4.4230510 & 0.2707440 & -0.9798460 \\ 23 & \mathrm{C} & -5.7116800 & -1.7312740 & 0.4737840 \\ 24 & \mathrm{C} & -5.7379200 & -0.0698270 & -1.2774300 \\ 25 & \mathrm{C} & -6.3876390 & -1.0715030 & -0.5528100 \\ 26 & \mathrm{H} & 2.2301850 & 4.5113090 & 0.4037360 \\ 27 & \mathrm{H} & 1.8595470 & 4.2467910 & -1.2955220 \\ 28 & \mathrm{H} & 3.6692320 & 2.6064550 & 0.4125120 \\ 29 & \mathrm{H} & 3.4619930 & 2.4876240 & -1.3236060 \\ 30 & \mathrm{H} & 4.4630880 & 0.3939310 & 0.9187570 \\ 31 & \mathrm{H} & -0.0307350 & -0.5481810 & 3.4745590 \\ 32 & \mathrm{H} & 0.7394270 & -1.6437850 & 2.3186320 \\ 33 & \mathrm{H} & 1.3542680 & 0.0034460 & 2.5143860 \\ 34 & \mathrm{H} & 1.0792880 & -1.7008900 & -2.7818520 \\ 35 & \mathrm{H} & 0.5344490 & -0.1508460 & -2.1286950 \\ 36 & \mathrm{H} & 0.0761260 & -1.6557460 & -1.3316200 \\ 37 & \mathrm{H} & 6.2118830 & -2.6105450 & -0.2296000 \\ 38 & \mathrm{H} & 6.1766430 & -1.5352540 & 1.1741860 \\ 39 & \mathrm{H} & 5.3591440 & -3.1022650 & 1.2343020 \\ 40 & \mathrm{H} & -3.8749720 & -1.9114360 & 1.5751510 \\ 41 & \mathrm{H} & -3.9176140 & 1.0479470 & -1.5392900 \\ 43 & \mathrm{H} & -6.2099130 & -2.5096540 & 1.0402030 \\ \mathrm{H} & -6.2581430 & 0.4473880 & -2.0754860 \\ \mathrm{H} & -7.4127450 & -1.3346700 & -0.7867760\end{array}$


Cartesian coordinate columns of the optimized structure for compound $\mathbf{3 c}$

(B3LYP/6-311+G(d,p)/PCM(acetonitrile))

$\begin{array}{lllll}1 & \mathrm{C} & -0.9978740 & 3.3593480 & -0.0506100 \\ 2 & \mathrm{C} & -2.4688220 & 3.7399100 & -0.2378460 \\ 3 & \mathrm{C} & -0.9378680 & 1.8980460 & 0.0960620 \\ 4 & \mathrm{C} & -3.2951730 & 2.4711370 & 0.0725300 \\ 5 & \mathrm{C} & -2.2850170 & 1.3416510 & 0.0139820 \\ 6 & \mathrm{C} & 0.1188110 & 1.0545590 & 0.2660150 \\ 7 & \mathrm{C} & -0.1525710 & -0.4192440 & 0.5725660 \\ 8 & \mathrm{~N} & 1.4700510 & 1.2470270 & 0.1518150 \\ 9 & \mathrm{O} & 1.1566190 & -0.9943400 & 0.2478050 \\ 10 & \mathrm{C} & 2.0056850 & 0.0519450 & 0.1425460 \\ 11 & \mathrm{C} & -2.4913380 & 0.0133550 & -0.1510230 \\ 12 & \mathrm{C} & -3.7310440 & -0.7199890 & -0.2054360 \\ 13 & \mathrm{C} & -1.2752680 & -0.9208590 & -0.3409270 \\ 14 & \mathrm{C} & -3.6183840 & -2.0629290 & -0.1002370 \\ 15 & \mathrm{~S} & -1.9346400 & -2.6235310 & 0.0844430 \\ 16 & \mathrm{O} & -0.0708730 & 4.1565150 & -0.0312910 \\ 17 & \mathrm{C} & -0.3781290 & -0.6265420 & 2.0776910 \\ 18 & \mathrm{C} & -0.8603320 & -0.9341060 & -1.8291360 \\ 19 & \mathrm{C} & 3.4262490 & -0.2413690 & 0.0080080 \\ 20 & \mathrm{C} & 3.8859910 & -1.5680780 & 0.0205500 \\ 21 & \mathrm{C} & 4.3443770 & 0.8126490 & -0.1349830 \\ 22 & \mathrm{C} & 5.2454660 & -1.8329330 & -0.1066230 \\ 23 & \mathrm{C} & 5.7003680 & 0.5390570 & -0.2606860 \\ 24 & \mathrm{C} & 6.1536430 & -0.7826500 & -0.2471250 \\ 25 & \mathrm{C} & -4.7146610 & -3.0774660 & -0.1074040 \\ 26 & \mathrm{H} & -2.7197610 & 4.5991950 & 0.3865350 \\ 27 & \mathrm{H} & -2.5933340 & 4.0520520 & -1.2807420 \\ 28 & \mathrm{H} & -3.7282330 & 2.5363630 & 1.0767610 \\ 29 & \mathrm{H} & -4.1220970 & 2.3286500 & -0.6251910 \\ 30 & \mathrm{H} & -4.6954030 & -0.2318100 & -0.2835390 \\ 31 & \mathrm{H} & 0.4268330 & -0.1464230 & 2.6370130 \\ 32 & \mathrm{H} & -1.3276420 & -0.1873230 & 2.3838770 \\ 33 & \mathrm{H} & -0.3907140 & -1.6897240 & 2.3155500 \\ 34 & \mathrm{H} & -0.5428340 & 0.0616740 & -2.1489030 \\ 35 & \mathrm{H} & -0.0429590 & -1.6353100 & -2.0014460 \\ 36 & \mathrm{H} & -1.7126980 & -1.2322940 & -2.4401140 \\ 37 & \mathrm{H} & 3.1796120 & -2.3802330 & 0.1321160 \\ 38 & \mathrm{H} & 3.9830320 & 1.8328690 & -0.1459810 \\ 39 & \mathrm{H} & 5.5971610 & -2.8577410 & -0.0954170 \\ 40 & \mathrm{H} & 6.4061160 & 1.3539590 & -0.3700190 \\ 41 & \mathrm{H} & 7.2125000 & -0.9923620 & -0.3455780 \\ 42 & \mathrm{H} & -4.6999660 & -3.6840940 & 0.8038250 \\ 44 & \mathrm{H} & -5.6862960 & -2.5856330 & -0.1790780 \\ & & -4.6100430 & -3.7648670 & -0.9530670\end{array}$


Cartesian coordinate columns of the optimized structure for compound $\mathbf{4 0}$

(B3LYP/6-311+G(d,p)/PCM(acetonitrile))

\begin{tabular}{|c|c|c|c|c|}
\hline 1 & C & -1.0285820 & 3.4135860 & 0.0033220 \\
\hline 2 & $\mathrm{C}$ & 0.1700660 & 4.2671570 & -0.3840290 \\
\hline 3 & $\mathrm{C}$ & -0.6023960 & 1.9940830 & 0.0106530 \\
\hline 4 & $\mathrm{C}$ & 1.3446020 & 3.2865210 & -0.4816230 \\
\hline 5 & $\mathrm{C}$ & 0.7235030 & 1.9189830 & -0.2861620 \\
\hline 6 & $\mathrm{C}$ & -1.5250390 & 0.9215300 & 0.3960470 \\
\hline 7 & $\mathrm{C}$ & -1.4380980 & 0.0597290 & 1.4491030 \\
\hline 8 & $\mathrm{~N}$ & -2.7169050 & 0.6817460 & -0.2889660 \\
\hline 9 & $\mathrm{O}$ & -2.5637990 & -0.7276270 & 1.4191200 \\
\hline 10 & $\mathrm{C}$ & -3.2886650 & -0.2967260 & 0.3464680 \\
\hline 11 & $\mathrm{C}$ & 1.5661030 & 0.7399660 & -0.4217480 \\
\hline 12 & $\mathrm{~N}$ & 2.9127170 & 0.7920120 & -0.0508270 \\
\hline 13 & $\mathrm{C}$ & 1.3038950 & -0.4949280 & -0.9553960 \\
\hline 14 & $\mathrm{C}$ & 3.3963460 & -0.3771100 & -0.3336950 \\
\hline 15 & $\mathrm{O}$ & 2.4695260 & -1.2098690 & -0.8959270 \\
\hline 16 & $\mathrm{O}$ & -2.1385500 & 3.8385300 & 0.2830660 \\
\hline 17 & $\mathrm{C}$ & -0.4627800 & -0.1732620 & 2.5401060 \\
\hline 18 & $\mathrm{C}$ & 0.1474180 & -1.1570050 & -1.6024800 \\
\hline 19 & $\mathrm{C}$ & 4.7508270 & -0.8818430 & -0.1292730 \\
\hline 20 & $\mathrm{C}$ & 5.7249330 & -0.0385250 & 0.4275180 \\
\hline 21 & $\mathrm{C}$ & 5.0949770 & -2.1961200 & -0.4785870 \\
\hline 22 & $\mathrm{C}$ & 7.0190170 & -0.5047560 & 0.6296080 \\
\hline 23 & $\mathrm{C}$ & 6.3930410 & -2.6556660 & -0.2726160 \\
\hline 24 & $\mathrm{C}$ & 7.3579800 & -1.8140900 & 0.2810000 \\
\hline 25 & $\mathrm{C}$ & -4.5581910 & -0.9571670 & 0.0554470 \\
\hline 26 & $\mathrm{C}$ & -5.0255030 & -2.0105730 & 0.8556250 \\
\hline 27 & $\mathrm{C}$ & -5.3257440 & -0.5335620 & -1.0409740 \\
\hline 28 & $\mathrm{C}$ & -6.2384720 & -2.6275250 & 0.5613210 \\
\hline 29 & $\mathrm{C}$ & -6.5360260 & -1.1549420 & -1.3287410 \\
\hline 30 & $\mathrm{C}$ & -6.9971750 & -2.2036500 & -0.5298480 \\
\hline 31 & $\mathrm{H}$ & 0.3140620 & 5.0562850 & 0.3570150 \\
\hline 32 & $\mathrm{H}$ & -0.0404130 & 4.7561030 & -1.3394620 \\
\hline 33 & $\mathrm{H}$ & 2.1006790 & 3.4615950 & 0.2888450 \\
\hline 34 & $\mathrm{H}$ & 1.8651610 & 3.3331060 & -1.4417610 \\
\hline 35 & $\mathrm{H}$ & 0.3037060 & 0.6013640 & 2.5229250 \\
\hline 36 & $\mathrm{H}$ & -0.9577600 & -0.1513620 & 3.5148780 \\
\hline 37 & $\mathrm{H}$ & 0.0285010 & -1.1456430 & 2.4345610 \\
\hline 38 & $\mathrm{H}$ & -0.2797440 & -1.9297760 & -0.9555670 \\
\hline 39 & $\mathrm{H}$ & 0.4636200 & -1.6370070 & -2.5321840 \\
\hline 40 & $\mathrm{H}$ & -0.6315070 & -0.4316440 & -1.8290930 \\
\hline 41 & $\mathrm{H}$ & 5.4582520 & 0.9756610 & 0.6974160 \\
\hline 42 & $\mathrm{H}$ & 4.3495110 & -2.8532740 & -0.9083070 \\
\hline 43 & $\mathrm{H}$ & 7.7650720 & 0.1531720 & 1.0600940 \\
\hline 44 & $\mathrm{H}$ & 6.6503750 & -3.6726690 & -0.5449930 \\
\hline 45 & $\mathrm{H}$ & 8.3674910 & -2.1750530 & 0.4403340 \\
\hline 46 & $\mathrm{H}$ & -4.4410200 & -2.3434040 & 1.7040800 \\
\hline 47 & $\mathrm{H}$ & -4.9664680 & 0.2804790 & -1.6580680 \\
\hline 48 & $\mathrm{H}$ & -6.5910200 & -3.4404820 & 1.1855370 \\
\hline 49 & $\mathrm{H}$ & -7.1217490 & -0.8208200 & -2.1774240 \\
\hline 50 & $\mathrm{H}$ & -7.9410600 & -2.6858400 & -0.7566150 \\
\hline
\end{tabular}


Cartesian coordinate columns of the optimized structure for compound $\mathbf{4 c}$

(B3LYP/6-311+G(d,p)/PCM(acetonitrile))

$\begin{array}{lllll}1 & \mathrm{C} & 1.2818750 & 3.7639540 & 0.0147000 \\ 2 & \mathrm{C} & 0.0913840 & 4.7117750 & -0.1629430 \\ 3 & \mathrm{C} & 0.7456230 & 2.3945350 & 0.0799360 \\ 4 & \mathrm{C} & -1.1832970 & 3.8749300 & 0.0957310 \\ 5 & \mathrm{C} & -0.7120180 & 2.4443060 & -0.0358830 \\ 6 & \mathrm{C} & 1.3650200 & 1.1915270 & 0.2493490 \\ 7 & \mathrm{C} & 0.5241180 & -0.0734950 & 0.4797890 \\ 8 & \mathrm{~N} & 2.6861080 & 0.8352460 & 0.1867530 \\ 9 & \mathrm{O} & 1.5130420 & -1.1028070 & 0.1534690 \\ 10 & \mathrm{C} & 2.7095260 & -0.4724480 & 0.1317790 \\ 11 & \mathrm{C} & -1.3922880 & 1.3020820 & -0.2494270 \\ 12 & \mathrm{~N} & -2.7585800 & 1.0487200 & -0.2228620 \\ 13 & \mathrm{C} & -0.6654750 & -0.0337640 & -0.4663250 \\ 14 & \mathrm{C} & -2.8871040 & -0.2382850 & -0.1387390 \\ 15 & \mathrm{O} & -1.7303980 & -0.9704120 & -0.1136090 \\ 16 & \mathrm{O} & 2.4485610 & 4.1195620 & 0.0907800 \\ 17 & \mathrm{C} & 0.1493330 & -0.2475720 & 1.9578920 \\ 18 & \mathrm{C} & -0.3003770 & -0.2709780 & -1.9365650 \\ 19 & \mathrm{C} & 3.9059500 & -1.2963250 & 0.0298000 \\ 20 & \mathrm{C} & 3.8100590 & -2.6969990 & 0.0058260 \\ 21 & \mathrm{C} & 5.1676210 & -0.6823120 & -0.0452740 \\ 22 & \mathrm{C} & 4.9626700 & -3.4694000 & -0.0903620 \\ 23 & \mathrm{C} & 6.3132960 & -1.4614250 & -0.1414590 \\ 24 & \mathrm{C} & 6.2136330 & -2.8549770 & -0.1641520 \\ 25 & \mathrm{C} & -4.1425420 & -0.9789140 & -0.0443920 \\ 26 & \mathrm{C} & -5.3629900 & -0.2836150 & -0.0244310 \\ 27 & \mathrm{C} & -4.1439000 & -2.3801470 & 0.0303260 \\ 28 & \mathrm{C} & -6.5607470 & -0.9814190 & 0.0672280 \\ 29 & \mathrm{C} & -5.3483100 & -3.0723750 & 0.1229420 \\ 30 & \mathrm{C} & -6.5572640 & -2.3770500 & 0.1415150 \\ 31 & \mathrm{H} & 0.1965500 & 5.5784460 & 0.4915990 \\ 32 & \mathrm{H} & 0.1216740 & 5.0798810 & -1.1946440 \\ 33 & \mathrm{H} & -1.5582260 & 4.0569040 & 1.1087280 \\ 34 & \mathrm{H} & -7.4992090 & -0.4395370 & 0.0818080 \\ 50 & \mathrm{H} & -5.3427160 & -4.1546030 & 0.1797150 \\ 34 & \mathrm{H} & -1.9939970 & 4.1097360 & -0.5951210 \\ 43 & \mathrm{H} & 1.0490170 & -0.1953180 & 2.5725390 \\ 36 & \mathrm{H} & -0.5356160 & 0.5389330 & 2.2754880 \\ 37 & \mathrm{H} & -0.3302160 & -1.2152940 & 2.1061350 \\ 38 & \mathrm{H} & 0.0865770 & -1.2814180 & -2.0740760 \\ 39 & \mathrm{H} & -1.1893110 & -0.1429100 & -2.5559710 \\ 40 & \mathrm{H} & 0.4542900 & 0.4446500 & -2.2675860 \\ 41 & \mathrm{H} & 2.8386850 & -3.1701810 & 0.0658510 \\ \mathrm{H} & \mathrm{H} & 4.2334230 & 0.3978550 & -0.0284800 \\ 43 & 7.2849490 & -0.9854630 & -0.1994770 \\ 47 & -5.3578720 & 0.7974750 & -0.0805670 \\ 43 & & & -2.9183650 & 0.2135230\end{array}$


Cartesian coordinate columns of the optimized structure for compound $\mathbf{5 0}$

(B3LYP/6-311+G(d,p)/PCM(acetonitrile))

\begin{tabular}{|c|c|c|c|c|}
\hline 1 & $\mathrm{C}$ & -4.7287790 & 2.4927050 & 0.1370500 \\
\hline 2 & $\mathrm{C}$ & -3.8307860 & 3.6978010 & -0.0978250 \\
\hline 3 & $\mathrm{C}$ & -3.9079680 & 1.2645010 & 0.0252820 \\
\hline 4 & $\mathrm{C}$ & -2.4225850 & 3.1181140 & -0.2691060 \\
\hline 5 & $\mathrm{C}$ & -2.6183540 & 1.6154310 & -0.2460740 \\
\hline 6 & $\mathrm{C}$ & -4.4974180 & -0.0654720 & 0.2609560 \\
\hline 7 & $\mathrm{C}$ & -4.0491910 & -0.9672570 & 1.1968190 \\
\hline 8 & $\mathrm{C}$ & -5.6648170 & -0.5310400 & -0.4434530 \\
\hline 9 & $\mathrm{~S}$ & -5.0527680 & -2.4013730 & 1.1893290 \\
\hline 10 & $\mathrm{C}$ & -6.0880090 & -1.7724980 & -0.0768830 \\
\hline 11 & $\mathrm{C}$ & -1.4701210 & 0.7608950 & -0.5053440 \\
\hline 12 & $\mathrm{~N}$ & -0.1874390 & 1.1635900 & -0.1139050 \\
\hline 13 & $\mathrm{C}$ & -1.3732500 & -0.4243080 & -1.1828670 \\
\hline 14 & $\mathrm{C}$ & 0.6131710 & 0.2270140 & -0.5264820 \\
\hline 15 & $\mathrm{O}$ & -0.0463060 & -0.7706010 & -1.1954680 \\
\hline 16 & $\mathrm{O}$ & -5.9243250 & 2.5456530 & 0.3892800 \\
\hline 17 & $\mathrm{C}$ & -2.9014790 & -0.8622810 & 2.1538900 \\
\hline 18 & $\mathrm{C}$ & -2.3027710 & -1.3206780 & -1.9093750 \\
\hline 19 & $\mathrm{C}$ & -7.2457990 & -2.5644500 & -0.6039510 \\
\hline 20 & $\mathrm{C}$ & 2.0524200 & 0.1133720 & -0.3707160 \\
\hline 21 & $\mathrm{C}$ & 2.7678600 & 1.0834440 & 0.3506250 \\
\hline 22 & $\mathrm{C}$ & 2.7749880 & -0.9544010 & -0.9261390 \\
\hline 23 & $\mathrm{C}$ & 4.1388510 & 0.9950270 & 0.5142150 \\
\hline 24 & $\mathrm{C}$ & 4.1485090 & -1.0517610 & -0.7749500 \\
\hline 25 & $\mathrm{C}$ & 4.8768890 & -0.0786280 & -0.0466300 \\
\hline 26 & $\mathrm{~N}$ & 6.2378390 & -0.1724170 & 0.1116940 \\
\hline 27 & $\mathrm{C}$ & 7.0372450 & 0.9294970 & 0.6514290 \\
\hline 28 & $\mathrm{C}$ & 6.9806610 & -1.3763700 & -0.2652360 \\
\hline 29 & $\mathrm{C}$ & 8.4545710 & 0.8614600 & 0.0957880 \\
\hline 30 & $\mathrm{C}$ & 8.2483010 & -1.4862890 & 0.5727950 \\
\hline 31 & $\mathrm{O}$ & 9.1254090 & -0.3639840 & 0.4150270 \\
\hline 32 & $\mathrm{H}$ & -4.1739310 & 4.2251890 & -0.9923810 \\
\hline 33 & $\mathrm{H}$ & -3.9231900 & 4.3927390 & 0.7399280 \\
\hline 34 & $\mathrm{H}$ & -1.9432990 & 3.4260510 & -1.2023050 \\
\hline 35 & $\mathrm{H}$ & -1.7464130 & 3.4160140 & 0.5371670 \\
\hline 36 & $\mathrm{H}$ & -6.1613970 & 0.0518410 & -1.2088680 \\
\hline 37 & $\mathrm{H}$ & -2.0204970 & -1.4030530 & 1.7922100 \\
\hline 38 & $\mathrm{H}$ & -2.6154630 & 0.1823670 & 2.2877270 \\
\hline 39 & $\mathrm{H}$ & -3.1603140 & -1.2715660 & 3.1335390 \\
\hline 40 & $\mathrm{H}$ & -3.2428860 & -0.8114700 & -2.1127430 \\
\hline 41 & $\mathrm{H}$ & -1.8576610 & -1.6342400 & -2.8570350 \\
\hline 42 & $\mathrm{H}$ & -2.5252360 & -2.2201010 & -1.3261890 \\
\hline 43 & $\mathrm{H}$ & -6.9231320 & -3.5104320 & -1.0489430 \\
\hline 44 & $\mathrm{H}$ & -7.9680730 & -2.8018410 & 0.1828020 \\
\hline 45 & $\mathrm{H}$ & -7.7636530 & -1.9889940 & -1.3739240 \\
\hline 46 & $\mathrm{H}$ & 2.2333180 & 1.9121520 & 0.7992070 \\
\hline 47 & $\mathrm{H}$ & 2.2563890 & -1.7159830 & -1.4957390 \\
\hline 48 & $\mathrm{H}$ & 4.6389450 & 1.7524570 & 1.1018330 \\
\hline 49 & $\mathrm{H}$ & 4.6618030 & -1.8793190 & -1.2446340 \\
\hline 50 & $\mathrm{H}$ & 6.6082960 & 1.8849730 & 0.3420880 \\
\hline 51 & $\mathrm{H}$ & 7.0555340 & 0.9136080 & 1.7494600 \\
\hline 52 & $\mathrm{H}$ & 6.3752890 & -2.2629780 & -0.0641580 \\
\hline 53 & $\mathrm{H}$ & 7.2281520 & -1.3789590 & -1.3354450 \\
\hline 54 & $\mathrm{H}$ & 8.4253630 & 0.9878950 & -0.9934950 \\
\hline 55 & $\mathrm{H}$ & 9.0537900 & 1.6703370 & 0.5173700 \\
\hline 56 & $\mathrm{H}$ & 7.9779600 & -1.5917670 & 1.6307170 \\
\hline 57 & $\mathrm{H}$ & 8.8107610 & -2.3726950 & 0.2744000 \\
\hline
\end{tabular}


Cartesian coordinate columns of the optimized structure for compound $\mathbf{5 c}$

(B3LYP/6-311+G(d,p)/PCM(acetonitrile))

\begin{tabular}{|c|c|c|c|c|}
\hline 1 & $\mathrm{C}$ & -5.2260280 & 2.1710940 & -0.0649800 \\
\hline 2 & $\mathrm{C}$ & -4.6080370 & 3.5649230 & 0.0734260 \\
\hline 3 & $\mathrm{C}$ & -4.1294580 & 1.1931210 & -0.0557510 \\
\hline 4 & $\mathrm{C}$ & -3.0810240 & 3.3886920 & -0.1036010 \\
\hline 5 & $\mathrm{C}$ & -2.8622110 & 1.9018200 & 0.0718250 \\
\hline 6 & $\mathrm{C}$ & -4.1954220 & -0.1682480 & -0.2042230 \\
\hline 7 & $\mathrm{C}$ & -2.8721480 & -0.9383970 & -0.3733890 \\
\hline 8 & $\mathrm{C}$ & -5.3313520 & -1.0285140 & -0.2545110 \\
\hline 9 & $\mathrm{~S}$ & -3.3268570 & -2.7193630 & -0.0129320 \\
\hline 10 & $\mathrm{C}$ & -5.0497980 & -2.3577810 & -0.1683210 \\
\hline 11 & $\mathrm{C}$ & -1.7450980 & 1.1845970 & 0.2985240 \\
\hline 12 & $\mathrm{~N}$ & -0.4075300 & 1.5463910 & 0.2714860 \\
\hline 13 & $\mathrm{C}$ & -1.8327430 & -0.3207560 & 0.5698620 \\
\hline 14 & $\mathrm{C}$ & 0.2710950 & 0.4343180 & 0.2385670 \\
\hline 15 & $\mathrm{O}$ & -0.4642430 & -0.7233140 & 0.2531800 \\
\hline 16 & $\mathrm{O}$ & -6.4268680 & 1.9464000 & -0.1614420 \\
\hline 17 & $\mathrm{C}$ & -2.4393610 & -0.8653160 & -1.8587630 \\
\hline 18 & $\mathrm{C}$ & -2.0629310 & -0.6003940 & 2.0615830 \\
\hline 19 & $\mathrm{C}$ & -6.0282800 & -3.4854340 & -0.1778850 \\
\hline 20 & $\mathrm{C}$ & 1.7098110 & 0.3003130 & 0.1642470 \\
\hline 21 & $\mathrm{C}$ & 2.5338330 & 1.4407180 & 0.1262690 \\
\hline 22 & $\mathrm{C}$ & 2.3320060 & -0.9601390 & 0.1265380 \\
\hline 23 & $\mathrm{C}$ & 3.9084720 & 1.3337080 & 0.0570480 \\
\hline 24 & $\mathrm{C}$ & 3.7079740 & -1.0820200 & 0.0536620 \\
\hline 25 & $\mathrm{C}$ & 4.5442180 & 0.0634260 & 0.0180070 \\
\hline 26 & $\mathrm{~N}$ & 5.9072920 & -0.0495180 & -0.0500410 \\
\hline 27 & $\mathrm{C}$ & 6.7735210 & 1.1019490 & -0.3188530 \\
\hline 28 & $\mathrm{C}$ & 6.5918410 & -1.3313810 & 0.1410810 \\
\hline 29 & $\mathrm{C}$ & 8.0555840 & 0.6373480 & -0.9988470 \\
\hline 30 & $\mathrm{C}$ & 8.0006230 & -1.0916870 & 0.6694180 \\
\hline 31 & $\mathrm{O}$ & 8.8034860 & -0.2976170 & -0.2119630 \\
\hline 32 & $\mathrm{H}$ & -5.0629700 & 4.2523910 & -0.6421310 \\
\hline 33 & $\mathrm{H}$ & -4.8543510 & 3.9344590 & 1.0750790 \\
\hline 34 & $\mathrm{H}$ & -2.7737970 & 3.7058380 & -1.1061380 \\
\hline 35 & $\mathrm{H}$ & -2.5038150 & 3.9791900 & 0.6098240 \\
\hline 36 & $\mathrm{H}$ & -6.3427080 & -0.6490670 & -0.3069440 \\
\hline 37 & $\mathrm{H}$ & -3.2329940 & -1.2628550 & -2.4918560 \\
\hline 38 & $\mathrm{H}$ & -2.2475240 & 0.1692970 & -2.1498870 \\
\hline 39 & $\mathrm{H}$ & -1.5327320 & -1.4484460 & -2.0203100 \\
\hline 40 & $\mathrm{H}$ & -3.0688650 & -0.2984950 & 2.3584120 \\
\hline 41 & $\mathrm{H}$ & -1.9354160 & -1.6601840 & 2.2828420 \\
\hline 42 & $\mathrm{H}$ & -1.3430050 & -0.0284810 & 2.6500730 \\
\hline 43 & $\mathrm{H}$ & -5.8579780 & -4.1398060 & -1.0386830 \\
\hline 44 & $\mathrm{H}$ & -5.9275370 & -4.1026490 & 0.7200840 \\
\hline 45 & $\mathrm{H}$ & -7.0492470 & -3.1045450 & -0.2264210 \\
\hline 46 & $\mathrm{H}$ & 2.0775450 & 2.4222870 & 0.1632840 \\
\hline 47 & $\mathrm{H}$ & 1.7243470 & -1.8561200 & 0.1458200 \\
\hline 48 & $\mathrm{H}$ & 4.4990740 & 2.2392740 & 0.0570720 \\
\hline 49 & $\mathrm{H}$ & 4.1376340 & -2.0727190 & 0.0019260 \\
\hline 50 & $\mathrm{H}$ & 6.2711110 & 1.7915780 & -0.9998610 \\
\hline 51 & $\mathrm{H}$ & 7.0051180 & 1.6524730 & 0.6022980 \\
\hline 52 & $\mathrm{H}$ & 6.0575990 & -1.9285880 & 0.8828950 \\
\hline 53 & $\mathrm{H}$ & 6.6258260 & -1.9100830 & -0.7913290 \\
\hline 54 & $\mathrm{H}$ & 7.8113490 & 0.1848060 & -1.9677710 \\
\hline 55 & $\mathrm{H}$ & 8.7064880 & 1.4949050 & -1.1776820 \\
\hline 56 & $\mathrm{H}$ & 7.9450950 & -0.6051760 & 1.6510480 \\
\hline 57 & $\mathrm{H}$ & 8.5136260 & -2.0470960 & 0.7913470 \\
\hline
\end{tabular}


Cartesian coordinate columns of the optimized structure for compound $\mathbf{6 0}$ (B3LYP/6-311+G(d,p)/PCM(acetonitrile))

$\begin{array}{lllll}1 & \mathrm{C} & 2.2557670 & -2.4405600 & -1.2066710 \\ 2 & \mathrm{C} & -2.6463310 & 2.6893380 & -0.1892660 \\ 3 & \mathrm{C} & -1.6176220 & 3.7768330 & -0.4588570 \\ 4 & \mathrm{C} & -1.9531210 & 1.3859020 & -0.1384150 \\ 5 & \mathrm{C} & -0.2696590 & 3.0492220 & -0.4819570 \\ 6 & \mathrm{C} & -0.6154140 & 1.5781970 & -0.3460890 \\ 7 & \mathrm{C} & -2.6964050 & 0.1532110 & 0.1819110 \\ 8 & \mathrm{C} & -2.4136350 & -0.6803260 & 1.2371530 \\ 9 & \mathrm{C} & -3.8569870 & -0.2683150 & -0.5605780 \\ 10 & \mathrm{~S} & -3.5643430 & -1.9995870 & 1.2983050 \\ 11 & \mathrm{C} & -4.4369140 & -1.4127170 & -0.1039150 \\ 12 & \mathrm{C} & 0.4617900 & 0.6074500 & -0.4395940 \\ 13 & \mathrm{~N} & 1.7336080 & 0.9563450 & -0.0177690 \\ 14 & \mathrm{C} & 0.4839190 & -0.6731570 & -0.9773010 \\ 15 & \mathrm{C} & 2.5076700 & -0.0810970 & -0.2618830 \\ 16 & \mathrm{~N} & 1.7868620 & -1.1024190 & -0.8486260 \\ 17 & \mathrm{O} & -3.8446580 & 2.8842590 & -0.0257080 \\ 18 & \mathrm{C} & -1.3273740 & -0.5871720 & 2.2645610 \\ 19 & \mathrm{C} & -0.5564290 & -1.4981970 & -1.6544530 \\ 20 & \mathrm{C} & 3.9462400 & -0.1268530 & 0.0548720 \\ 21 & \mathrm{C} & 4.3898610 & 0.3989950 & 1.2782770 \\ 22 & \mathrm{C} & 4.8954710 & -0.6274480 & -0.8489220 \\ 23 & \mathrm{C} & 5.7457390 & 0.4145220 & 1.5927050 \\ 24 & \mathrm{C} & 6.2521480 & -0.6125790 & -0.5300080 \\ 25 & \mathrm{C} & 6.6818620 & -0.0945660 & 0.6912190 \\ 26 & \mathrm{C} & -5.6364160 & -2.1358310 & -0.6369270 \\ 27 & \mathrm{H} & 3.1638500 & -2.6632180 & -0.6521660 \\ 28 & \mathrm{H} & 1.4961480 & -3.1739800 & -0.9396100 \\ 29 & \mathrm{H} & 2.4617440 & -2.5119510 & -2.2766680 \\ 30 & \mathrm{H} & -1.8482340 & 4.2551550 & -1.4150940 \\ 31 & \mathrm{H} & -1.6910840 & 4.5482810 & 0.3113920 \\ 32 & \mathrm{H} & 0.2949030 & 3.2225060 & -1.4018910 \\ 33 & \mathrm{H} & 0.3844530 & 3.3516310 & 0.3403590 \\ 34 & \mathrm{H} & -4.2344610 & 0.2729700 & -1.4190590 \\ 35 & \mathrm{H} & -0.4886380 & -1.2507870 & 2.0291290 \\ 36 & \mathrm{H} & -0.9385880 & 0.4314750 & 2.3107300 \\ 37 & \mathrm{H} & -1.6932580 & -0.8572270 & 3.2583740 \\ 38 & \mathrm{H} & -0.1523200 & -1.9863490 & -2.5450510 \\ 39 & \mathrm{H} & -0.9541750 & -2.2768490 & -0.9950990 \\ 40 & \mathrm{H} & -1.3920220 & -0.8720180 & -1.9597590 \\ 41 & \mathrm{H} & 3.6625770 & 0.7908270 & 1.9787710 \\ 43 & \mathrm{H} & 4.5823050 & -1.0046930 & -1.8148850 \\ \mathrm{H} & 6.0716920 & 0.8200140 & 2.5438600 \\ \mathrm{H} & 6.9734740 & -0.9969710 & -1.2421000 \\ 45 & -6.4346190 & -2.2045040 & 0.1082440 \\ 47 & -6.0309070 & -1.6025010 & -1.5042080\end{array}$


Cartesian coordinate columns of the optimized structure for compound $\mathbf{6 c}$

(B3LYP/6-311+G(d,p)/PCM(acetonitrile))

\begin{tabular}{|c|c|c|c|c|}
\hline 1 & C & 1.6383840 & -2.2544260 & 0.5614710 \\
\hline 2 & $\mathrm{C}$ & -3.1964940 & 2.4986470 & -0.1358240 \\
\hline 3 & $\mathrm{C}$ & -2.4152410 & 3.8138570 & -0.0797600 \\
\hline 4 & $\mathrm{C}$ & -2.2224390 & 1.3985020 & -0.0959010 \\
\hline 5 & $\mathrm{C}$ & -0.9248720 & 3.4483050 & -0.2756130 \\
\hline 6 & $\mathrm{C}$ & -0.8797340 & 1.9552420 & -0.0300170 \\
\hline 7 & $\mathrm{C}$ & -2.4528210 & 0.0499570 & -0.1721390 \\
\hline 8 & $\mathrm{C}$ & -1.2317550 & -0.8756030 & -0.3307510 \\
\hline 9 & $\mathrm{C}$ & -3.6839760 & -0.6696230 & -0.1653550 \\
\hline 10 & S & -1.8901700 & -2.5737360 & 0.1293640 \\
\hline 11 & $\mathrm{C}$ & -3.5624140 & -2.0172590 & -0.0212560 \\
\hline 12 & $\mathrm{C}$ & 0.1601840 & 1.1300520 & 0.2101830 \\
\hline 13 & $\mathrm{~N}$ & 1.5219100 & 1.3752100 & 0.1365480 \\
\hline 14 & $\mathrm{C}$ & -0.0863640 & -0.3483670 & 0.5571750 \\
\hline 15 & $\mathrm{C}$ & 2.1100170 & 0.1970130 & 0.1478280 \\
\hline 16 & $\mathrm{~N}$ & 1.2650160 & -0.8802670 & 0.2505870 \\
\hline 17 & $\mathrm{O}$ & -4.4176260 & 2.4143950 & -0.1987210 \\
\hline 18 & $\mathrm{C}$ & -0.8483870 & -0.9243710 & -1.8306460 \\
\hline 19 & $\mathrm{C}$ & -0.3793570 & -0.4599050 & 2.0745770 \\
\hline 20 & $\mathrm{C}$ & -4.6676990 & -3.0196900 & 0.0319710 \\
\hline 21 & $\mathrm{C}$ & 3.5739500 & 0.0717660 & -0.0083890 \\
\hline 22 & $\mathrm{C}$ & 4.4114050 & 0.9581350 & 0.6839930 \\
\hline 23 & $\mathrm{C}$ & 4.1447560 & -0.8679500 & -0.8789960 \\
\hline 24 & $\mathrm{C}$ & 5.7923520 & 0.8937240 & 0.5218250 \\
\hline 25 & $\mathrm{C}$ & 5.5266300 & -0.9217840 & -1.0468860 \\
\hline 26 & $\mathrm{C}$ & 6.3538140 & -0.0465340 & -0.3433470 \\
\hline 27 & $\mathrm{H}$ & 2.7118170 & -2.3267100 & 0.7195460 \\
\hline 28 & $\mathrm{H}$ & 1.1422110 & -2.5888470 & 1.4747790 \\
\hline 29 & $\mathrm{H}$ & 1.3612510 & -2.9322040 & -0.2518270 \\
\hline 30 & $\mathrm{H}$ & -2.8039520 & 4.5162050 & -0.8196150 \\
\hline 31 & $\mathrm{H}$ & -2.5914350 & 4.2567880 & 0.9069390 \\
\hline 32 & $\mathrm{H}$ & -0.6057050 & 3.6807720 & -1.2977550 \\
\hline 33 & $\mathrm{H}$ & -0.2657090 & 3.9995910 & 0.3973270 \\
\hline 34 & $\mathrm{H}$ & -4.6440160 & -0.1747750 & -0.2232770 \\
\hline 35 & $\mathrm{H}$ & -1.7058850 & -1.2523570 & -2.4190620 \\
\hline 36 & $\mathrm{H}$ & -0.5473730 & 0.0662010 & -2.1772310 \\
\hline 37 & $\mathrm{H}$ & -0.0234360 & -1.6171500 & -1.9997050 \\
\hline 38 & $\mathrm{H}$ & -1.3111180 & 0.0542150 & 2.3139670 \\
\hline 39 & $\mathrm{H}$ & -0.4736180 & -1.4939200 & 2.4056520 \\
\hline 40 & $\mathrm{H}$ & 0.4298460 & 0.0162700 & 2.6316120 \\
\hline 41 & $\mathrm{H}$ & -4.5949990 & -3.7248240 & -0.8022730 \\
\hline 42 & $\mathrm{H}$ & -4.6243250 & -3.6073930 & 0.9541390 \\
\hline 43 & $\mathrm{H}$ & -5.6365430 & -2.5207510 & -0.0177430 \\
\hline 44 & $\mathrm{H}$ & 3.9717870 & 1.6906480 & 1.3494270 \\
\hline 45 & $\mathrm{H}$ & 3.5116590 & -1.5392310 & -1.4464830 \\
\hline 46 & $\mathrm{H}$ & 6.4302960 & 1.5778700 & 1.0694670 \\
\hline 47 & $\mathrm{H}$ & 5.9557040 & -1.6445210 & -1.7312050 \\
\hline 48 & $\mathrm{H}$ & 7.4291380 & -0.0939770 & -0.4715490 \\
\hline
\end{tabular}

